\title{
An Overview and Mechanism for the Coexistence of 5G NR-U (New Radio Unlicensed) in the Millimeter-Wave Spectrum for Indoor Small Cells
}

\author{
Rony Kumer Saha \\ Radio and Spectrum Laboratory, KDDI Research, Inc., 2-1-15 Ohara, Fujimino-shi, Saitama, Japan \\ Correspondence should be addressed to Rony Kumer Saha; rony107976@gmail.com
}

Received 10 April 2021; Revised 5 August 2021; Accepted 27 August 2021; Published 10 September 2021

Academic Editor: Giuseppe Piro

Copyright ( $) 2021$ Rony Kumer Saha. This is an open access article distributed under the Creative Commons Attribution License, which permits unrestricted use, distribution, and reproduction in any medium, provided the original work is properly cited.

\begin{abstract}
In this paper, we first give an overview of the coexistence of cellular with IEEE 802.11 technologies in the unlicensed bands. We then present a coexistence mechanism for Fifth-Generation (5G) New Radio on Unlicensed (NR-U) small cells located within buildings to coexist with the IEEE 802.11ad/ay, also termed as Wireless Gigabit (WiGig). Small cells are dual-band enabled operating in the $60 \mathrm{GHz}$ unlicensed and $28 \mathrm{GHz}$ licensed millimeter-wave $(\mathrm{mmW})$ bands. We develop an interference avoidance scheme in the time domain to avoid cochannel interference (CCI) between in-building NR-U small cells and WiGig access points (APs). We then derive average capacity, spectral efficiency (SE), and energy efficiency (EE) performance metrics of in-building small cells. Extensive system-level numerical and simulation results and analyses are carried out for a number of variants of NR-U, including NR standalone, NR-U standalone, and NR-U anchored. We also analyze the impact of the spatial reuse of both $\mathrm{mmW}$ spectra of multiple NR-U anchored operators with a WiGig operator. It is shown that NR-U anchored provides the best average capacity and EE performances, whereas NR-U standalone provides the best SE performance. Moreover, both vertical spatial reuse intrabuilding level and horizontal spatial reuse interbuilding level of mmW spectra in small cells of an NR-U anchored can improve its SE and EE performances. Finally, we show that by choosing appropriate values of vertical and horizontal spatial reuse factors, the proposed coexistence mechanism can achieve the expected SE and EE requirements for the future Sixth-Generation $(6 \mathrm{G})$ mobile networks.
\end{abstract}

\section{Introduction}

1.1. Background. The continuing demand for high capacity and data rate in cellular networks due to the growth of mobile devices to serve rich and diverse multimedia contents enforces mobile network operators (MNOs) to redesign the existing networks. Global mobile traffic is more than doubling each year [1], and in line with so, the introduction of the Fifth-Generation (5G) New Radio (NR) to serve a large volume of data traffic has increased the burden on the licensed spectrum bands of an MNO [2]. Serving such a large volume of data traffic in the unlicensed spectrum in addition to the licensed one of an MNO is an effective solution, and accordingly, the $3^{\text {rd }}$ Generation Partnership Project (3GPP) has recently started expanding the operation of cellular networks to unlicensed bands in 2015 with the Long-Term Evo- lution (LTE) in 3GPP Release-13 [3]. However, the IEEE 802.11-based Wireless Fidelity (WiFi) technologies have been developed and are in operation globally over a wide range of unlicensed bands, including $2.4 \mathrm{GHz}, 5 \mathrm{GHz}$, and $60 \mathrm{GHz}$ bands, since 1997 [4]. Hence, to operate in these unlicensed bands without interfering with each other, an appropriate mechanism is necessary for cellular networks to coexist with the incumbent WiFi networks.

1.2. Related Work. Numerous research studies addressed the coexistence of WiFi with cellular networks such as LTE and 5G NR. Notably, with regard to the LTE and WiFi (LTE/WiFi) coexistence, the authors in [3] elaborated LTE/WiFi coexistence mechanism in time, frequency, and power aspects. Further, the authors in [5] presented an analytical model for the characterization of achievable throughputs of 
WiFi and LTE on Unlicensed band (LTE-U) networks in spatially distributed scenarios in the downlink. Furthermore, the authors in [6] carried out a performance analysis of 3GPP LTE and IEEE 802.11 Wireless Local Area Networks (WLAN) using a fractional bandwidth sharing mechanism. Besides, the authors in [7] reviewed the state-of-the-art LTE/WiFi coexistence mechanisms and showed their incorporation into the industry standards.

Likewise, regarding the $5 \mathrm{G}$ New Radio Unlicensed (NR-U) and WiFi (5G NR-U/WiFi) coexistence, the authors in [8] addressed the coexistence of $5 \mathrm{G} \mathrm{NR}-\mathrm{U} / \mathrm{WiFi}$ in the $6 \mathrm{GHz}$ band, and in [9], the authors addressed the coexistence of WiFi with the beam-based $5 \mathrm{G}$ NR-U in the millimeter-wave (mmW) bands. Moreover, in [2], the authors investigated the performance of the $5 \mathrm{G}$ NR-U/WiFi coexisted network by implementing a mode selection procedure in $5 \mathrm{G}$ NR to use either the licensed spectrum band or the unlicensed spectrum band. Furthermore, the authors in [4] presented a system-level evaluation of $5 \mathrm{G}$ NR-U/WiFi coexistence in the $60 \mathrm{GHz}$ unlicensed mmW band. Likewise, in [10], the authors presented a system-level simulation-based study on the coexistence of NRbased access to unlicensed spectrum and an IEEE technology, i.e., Wireless Gigabit (WiGig), at $60 \mathrm{GHz}$ bands. Besides, in [11], the authors reported on extensions to a popular and open-source network simulator, ns-3, to build an NR-U system-level simulator and to model the coexistence of NR$\mathrm{U}$ and IEEE 802.11 technologies in the available unlicensed spectrum bands. Further, [12] examined the downlink performance of NR-U and WiGig technologies under intertechnology interference from each other in the $60 \mathrm{GHz}$ band. The author also presented stochastic models for signal-to-interference-plus-noise ratio (SINR) and data rate under a dense small cell setting.

Besides, several research studies proposed to use the almost blank subframe- (ABS-) based Enhanced Intercell Interference Coordination (eICIC) technique in LTE to address the coexistence issue between WiFi and cellular systems in the unlicensed band in the time-domain. For example, by reusing the concept of ABS, the authors in [13] proposed a simple scheme to exist the LTE system with the WiFi system in an unlicensed band and showed an improved throughput per WiFi user performance. Likewise, the authors in [14] proposed an adaptive coexistence scheme between LTE and WiFi by utilizing the ABS. Further, the authors in [13] proposed a modified version of ABSs called null subframes during which no reference signal is transmitted for reusing these blank subframes by the $\mathrm{WiFi}$ and showed that the throughput of WiFi increases with an increase in null subframes. Furthermore, the authors in [15] provided a performance evaluation of coexistence between LTE and WiFi systems and showed some of the challenges faced by each technology. Specifically, in an office scenario, the authors showed with simulation results that the LTE system performance is slightly affected by coexistence, whereas WiFi is impacted considerably. Likewise, the authors in [16] proposed to manage the coexistence between LTE-U and WiFi using an X2 interface where the ABSs are configured and communicated by LTE-U macrocells using X2 interface to allow WiFi data transmission during ABSs without any interruption from macrocells.

1.3. Problem Statement. Since existing MNOs are facing difficulty from the cost and scarcity of the available licensed spectrum, compared to the unlicensed spectrum available in the low-frequency bands, the unlicensed spectrum in the high-frequency $\mathrm{mmW}$ bands is a potential solution to address both the cost and scarcity issues for the 5G mobile networks. Particularly, due to the license-free access to the unlicensed band and wide spectrum bandwidth availability in the unlicensed, as well as licensed, $\mathrm{mmW}$ spectrum, both the spectrum licensing cost and scarcity can be minimized. However, to operate in unlicensed bands, certain regulatory requirements, including the use of Listen Before Talk (LBT) as a spectrum-sharing mechanism, a maximum channel occupancy time, a minimum occupied channel bandwidth requirement, and power limits [4], must be met. These regulatory requirements vary among regions and bands.

From Table 1, it can be found that most existing works investigated NR-U mainly under the LBT protocol as a native feature of NR-U. This, however, is not the case for the LTE since LBT has been introduced later to LTE, which has a different medium access control (MAC) mechanism from that of NR-U. Even though LBT is able to provide better fairness than other coexistence mechanisms, it suffers also from several bottlenecks. Firstly, LBT introduces extra delay due to the contention time overhead that leads to inefficient channel usage. Secondly, the use of LBT in LTE requires considerable changes in the original LTE MAC mechanism. Thirdly, the impact of IEEE 802.11 standards varies on how LBT is implemented since not all LBT schemes provide fair existence. Fourthly, if the duration of transmission bursts was too long, WiFi access points (APs) may get experienced large packet delays and jitter.

Moreover, LBT is not required by all regions in the world such as the USA, South Korea, and China. In such regions, more simple mechanisms such as blank subframes are preferable. In this regard, the allocation of air time between cellular and IEEE 802.11 nodes plays an important role in the fairness of unlicensed spectrum allocation and, hence, the overall coexistence performance. The major advantage of using blank subframes is that it requires fewer changes in the air interface of cellular standards such as LTE and hence can be deployed in a short term. For nonadjacent subframes, by reporting the duration and occurrence of blank subframes to WiFi APs during the negotiation phase, the transmission of WiFi APs can be suitably confined with the subframes to avoid interference with cellular nodes such as LTE.

Another noticeable factor is the operation of the highfrequency $\mathrm{mmW}$ spectrum. In this regard, a wider contiguous bandwidth is available in the $60 \mathrm{GHz}$ band, which is not currently very crowded. Due to these reasons, the $60 \mathrm{GHz}$ band is considered an attractive unlicensed band for $5 \mathrm{G}$ NR-U $[9,17]$. Moreover, the small coverage and high internal/external walls and floor penetration losses in the $60 \mathrm{GHz}$ unlicensed band make it suitable to operate with the small cells in indoor environments, particularly, in dense urban multistory buildings. 


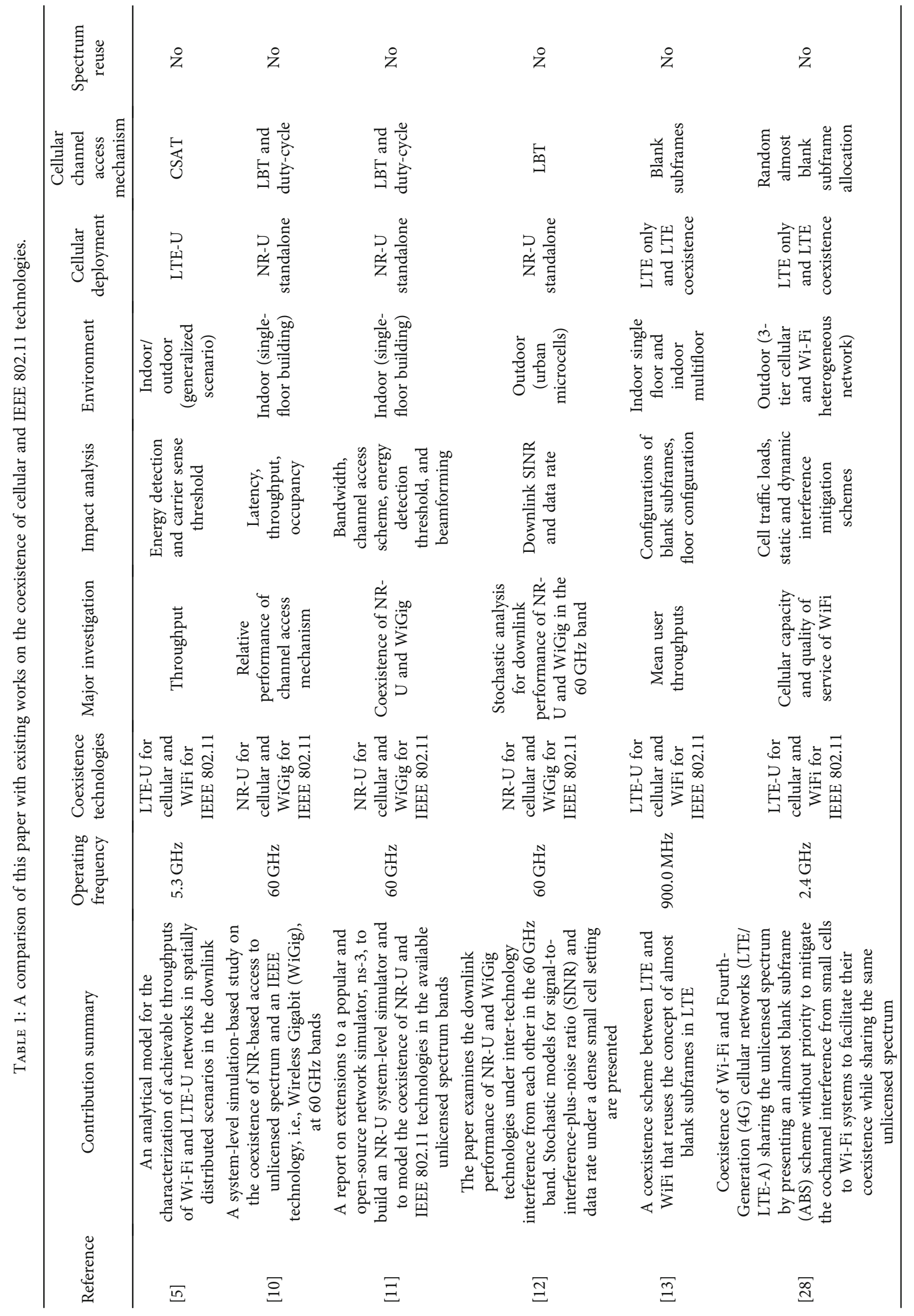




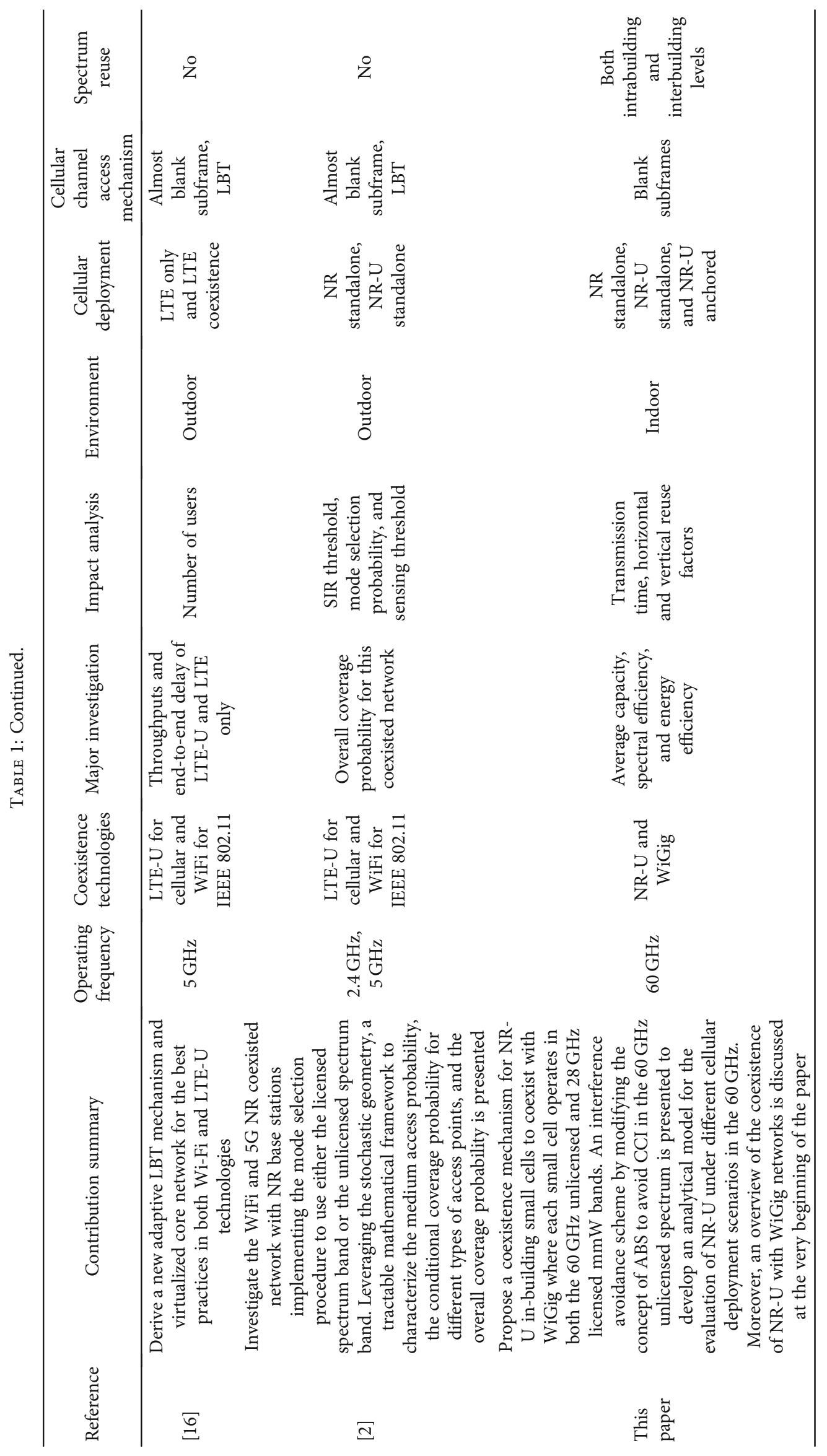


Besides, because of the regulatory restrictions on the transmission power in the unlicensed bands, like LTE Unlicensed [7], 5G NR-U is expected to be operated in the small cells deployed in the indoor coverage. Hence, as an extension of the LTE on Unlicensed bands or Licensed Assisted Access (LAA), 5G NR-U will aggregate the candidate $28 \mathrm{GHz}$ or $38 \mathrm{GHz} 5 \mathrm{G}$ licensed $\mathrm{mmW}$ spectrum and the $60 \mathrm{GHz}$ unlicensed $\mathrm{mmW}$ spectrum [17]. Therefore, NR is considered deploying in several scenarios such as NR standalone, NR-U standalone, and NR-U anchored. Furthermore, due to high floor and wall penetration losses in the $60 \mathrm{GHz}$ band, the same unlicensed spectrum can be reused more than once in small cells within a building, as well as adjacent buildings to serve the high data rate and capacity demands of indoor users.

However, research and investigation studies on the $5 \mathrm{G}$ NR-U operating in both the licensed and unlicensed $\mathrm{mmW}$ spectra for in-building small cells using non-LBT based coexistence mechanisms are in the early stage. Moreover, like [18], detailed mathematical modeling and analysis of major performance metrics, including average capacity, spectral efficiency (SE), and energy efficiency (EE), for 5G NR-U, are yet to be addressed, which we aim to contribute in this paper.

1.4. Contribution. Unlike existing studies, in this paper, following a brief overview of operating cellular technologies in the unlicensed bands, we propose a fully blank subframe(FBS-) based coexistence mechanism and derive optimal air time allocations to cellular/IEEE 802.11 nodes in terms of blank subframes for $5 \mathrm{G}$ NR-U operating in both the licensed and unlicensed $\mathrm{mmW}$ spectra for in-building small cells. We model and analyze major performance metrics, including average capacity, SE, EE, and spatial spectrum reuse of the same spectra more than once within each building, as well as between adjacent buildings, of small cells. Moreover, modeling and analyses are carried out for 5G NR-U under different deployment scenarios, including NR standalone, NR-U standalone, and NR-U anchored. The performance of the proposed mechanism is then justified against fulfilling the expected SE and EE requirements for the SixthGeneration (6G) mobile networks. More specifically, we contribute the following in this paper:

(i) We first give a brief overview, highlighting issues, challenges, and possible solution alternatives, for the coexistence of cellular and incumbent IEEE 802.11 technologies in the unlicensed bands

(ii) We then present an FBS-based coexistence mechanism and develop an interference avoidance scheme in time domain by modifying the concept of ABS for the LTE system to avoid cochannel interference (CCI) between in-building small cells of the 5G NR$\mathrm{U}$ operator and the incumbent WiGig operator in the $60 \mathrm{GHz}$ unlicensed $\mathrm{mmW}$ spectrum

(iii) We model traffic activity of in-building small cells and derive optimal number of FBSs, average capacity, SE, and EE responses of in-building 5G NR-U small cells coexisting with the IEEE 802.11ad/ay (i.e., WiGig) and operating in the $28 \mathrm{GHz}$ licensed and $60 \mathrm{GHz}$ unlicensed $\mathrm{mmW}$ bands

(iv) We carry out a system-level performance analysis for a number of variants of $5 \mathrm{G}$ NR-U, including 5G NR standalone that operates only in the $28 \mathrm{GHz}$ licensed $\mathrm{mmW}$ spectrum, 5G NR-U standalone that operates only in the $60 \mathrm{GHz}$ unlicensed $\mathrm{mmW}$ spectrum, and $5 \mathrm{G}$ NR-U anchored that operates in both the $28 \mathrm{GHz}$ licensed $\mathrm{mmW}$ spectrum and the $60 \mathrm{GHz}$ unlicensed $\mathrm{mmW}$ spectra

(v) We then analyze the impact of reusing unlicensed spectrum spatially within each building, as well as varying the number of $5 \mathrm{G} \mathrm{NR-U}$ operators

(vi) Finally, we compare the SE and EE performances of the proposed coexistence scheme against the expected $\mathrm{SE}$ and $\mathrm{EE}$ requirements for the future $6 \mathrm{G}$ mobile networks

To demonstrate the position of this paper in comparison with the existing works, Table 1 shows a comparison in the scope of a number of the existing works discussed in Section 1.2 with respect to this paper.

1.5. Organization. We organize the paper as follows. A brief overview, including key issues, challenges, and possible solutions, for the coexistence of a cellular network with an incumbent WiFi network in the unlicensed bands is given in Section 2. In Section 3, system architecture, coexistence mechanism, and CCI interference management are discussed. In Section 4, we model traffic activity of in-building small cells and derive average capacity, SE, and EE metrics for the 5G NR-U anchored, as well as 5G NR-U standalone. In Section 5, we carry out extensive system-level numerical and simulation results and analyses for a single NR-U operator, as well as multiple NR-U operators, by varying both the vertical and horizontal spatial spectrum reuse factors and compare the SE and EE performances of the proposed coexistence scheme against the expected SE and EE requirements for the future $6 \mathrm{G}$ mobile networks. We conclude the paper in Section 6. A list of abbreviations and a list of selected notations are given, respectively, in Tables 2 and 3.

\section{Coexistence of Cellular Networks in the Unlicensed Bands: An Overview}

Cellular technology is an allocation-based mechanism that uses continuous transmission of data in consecutive frames using a centralized scheduler located mainly in the macrocell BSs. However, WiFi technology is a contention-based mechanism that uses opportunistic transmission using distributed coordination function (DCF). DCF is a contention-based mechanism [19] that uses Carrier Sense Multiple Access with Collision Avoidance (CSMA/CA) protocol to detect the energy level to get access to a channel. In CSMA/CA [20], a WiFi AP senses the channel first to detect if any other WiFi AP is occupying the channel before sending its data to 
TABLE 2: A list of acronyms/abbreviations.

\begin{tabular}{|c|c|}
\hline $\begin{array}{l}\text { Acronym/ } \\
\text { abbreviation }\end{array}$ & Definition \\
\hline 3GPP & $3^{\text {rd }}$ Generation Partnership Project \\
\hline $5 G$ & Fifth-Generation \\
\hline 5G NR-U/WiFi & 5G New Radio Unlicensed and WiFi \\
\hline $6 \mathrm{G}$ & Sixth-Generation \\
\hline ABS & Almost blank subframe \\
\hline AP & Access point \\
\hline BS & Base station \\
\hline CCA & Clear channel assessment \\
\hline CCI & Cochannel interference \\
\hline COT & Channel occupancy time \\
\hline CSAT & Carrier Sense Adaptive Transmission \\
\hline CSMA/CA & $\begin{array}{c}\text { Carrier Sense Multiple Access with Collision } \\
\text { Avoidance }\end{array}$ \\
\hline DCF & Distributed coordination function \\
\hline $\mathrm{EE}$ & Energy efficiency \\
\hline eICIC & Enhanced Intercell Interference Coordination \\
\hline FBS & Fully blank subframe \\
\hline FPP & FBS pattern period \\
\hline hRF & Horizontal reuse factor \\
\hline LAA & Licensed assisted access \\
\hline LBT & Listen Before Talk \\
\hline LTE & Long-Term Evolution \\
\hline LTE/WiFi & LTE and WiFi \\
\hline LTE-U & LTE Unlicensed \\
\hline MAC & Medium access control \\
\hline MBS & Macrocell base station \\
\hline $\mathrm{mmW}$ & Millimeter-wave \\
\hline MNO & Mobile network operator \\
\hline NR Std & NR standalone \\
\hline NRA & National Regulatory Agency \\
\hline NR-U & New Radio on Unlicensed Band \\
\hline NR-U Anch & NR-U anchored \\
\hline NR-U Std & NR-U standalone \\
\hline PBS & Picocell base station \\
\hline $\mathrm{RB}$ & Resource block \\
\hline SBS & Small cell base station \\
\hline SE & Spectral efficiency \\
\hline TTI & Transmission time interval \\
\hline UE & User equipment \\
\hline vRF & Vertical reuse factor \\
\hline WiAP & Wireless Gigabit Access Point \\
\hline WiGig & Wireless Gigabit \\
\hline
\end{tabular}

avoid the collision with the existing occupant. If the WiFi AP finds that the channel is idle, it can start transmitting its data over the channel. Otherwise, it selects a random back-off timer so that it starts its transmission only when the timer decreases to zero [21]. Besides, the interference level of a cel- lular network is likely to be above the threshold used by a WiFi network to detect the vacancy of a channel.

Because of these disparities mentioned above in interference levels, as well as MAC layer procedures, of two systems (i.e., the absence of a contention-based mechanism like CSMA/CA to sense the channel status and the continuous transmission of data in a cellular network), if cellular and WiFi networks operate at the same frequency and location, WiFi APs may get blocked by a cellular network, leading the coexistence between them difficult to achieve. Since, due to employing CSMA/CA, a WiFi AP is used to back off its transmission when a channel is found busy, a cellular node as well should give up its transmission over the same channel to avoid any contention or collision with the WiFi AP. In other words, a cellular network needs to have a feature like CSMA/CA to avoid collision with a WiFi network to exist fairly with one another.

The 3GPP defines the fair coexistence between cellular networks such as LTE and WiFi (in the $5 \mathrm{GHz}$ ) as follows: the capability of an LAA network not to impact WiFi networks active on a carrier more than an additional WiFi network operating on the same carrier, in terms of throughput and latency $[22,23]$. Likewise, for $5 \mathrm{G} \mathrm{NR}-\mathrm{U}$, the coexistence requirement with WiGig remains the same as that in LAA such that the existence of an NR-U network cannot impact the performance of WiGig more than an additional WiGig network would do [24]. Hence, to ensure a fair coexistence of a $5 \mathrm{G}$ NR-U node with a WiGig AP in the unlicensed bands, an NR-U node needs to use a fair and efficient coexistence mechanism, called LBT [21]. Numerous research studies also showed that the LBT is critical for a fair coexistence between a cellular network and a WiFi network [25].

The LBT mechanism is fundamentally similar to the CSMA/CA mechanism; i.e., it does not allow a cellular node to always use a channel. Instead, it shares a channel between a cellular node and a WiFi AP on a fair basis [19]. In doing so, LBT enables a cellular node to periodically stop the occupancy of a channel and detect the activities of other shared nodes at a millisecond level. More specifically, to check the availability of a channel, a cellular node listens to the channel for a period called clear channel assessment (CCA) to detect the energy level. In this regard, an energy detection threshold is defined by the regional regulatory requirements to transmit over an unlicensed channel [26]. If the cellular node receives an energy level higher than the threshold level, the node considers that the channel is busy. However, if the energy level detected is below the threshold level, the cellular node can use the channel for a fixed time period, called channel occupancy time (COT). The idle period should be at least 5 percent of the COT [19], and the energy threshold level can be made adaptive, particularly in the downlink [21].

However, LBT is not required to be employed in all regions and all cellular standards. For example, in countries such as Europe and Japan, LBT features are mandatory for the $5 \mathrm{GHz}$ and $60 \mathrm{GHz}$ bands, whereas in other regions such as the United States and China, LBT is not required for early commercialization $[9,27]$. Similarly, unlike LTE-U, which is deployed in regions requiring no LBT feature, LBT is 
TABLE 3: A list of selected notations.

\begin{tabular}{|c|c|}
\hline Notation & Description \\
\hline$O_{\mathrm{M}}$ and $O_{\mathrm{W}}$ & Number of 5G NR-U operators and WiFi operators in a country \\
\hline$O$ and $L$ & Total number of operators and total number of buildings per macrocell, respectively \\
\hline$T_{o}$ and $T_{\mathrm{FPP}}$ & $\begin{array}{c}\text { An optimum value of the number of FBSs over } T_{\mathrm{FPP}} \text { of any operator } o \text { and FBS pattern period, } \\
\text { respectively }\end{array}$ \\
\hline$p(s)$ & Probability of $s$ number of active in-building stations of an operator $o$ on a floor $\mathrm{fl}$ in a building \\
\hline$\lambda_{o, s}$ & $\begin{array}{c}\text { The expected value of the number of in-building user equipments (UEs) of any operator } o \text { on a } \\
\text { floor } \mathrm{fl} \text { within a building }\end{array}$ \\
\hline$W_{\mathrm{AP}, o}$ & Number of WiAPs of WiGig operator $o$ on each floor of any building \\
\hline$S_{\mathrm{F}, o}$ and $S_{\mathrm{F}, \text { total }}$ & Number of small cell base stations of 5G NR-U operator $o$ per floor and per building, respectively \\
\hline$Q$ and $\varepsilon_{\mathrm{RF}}$ & Maximum simulation run time and vertical spectrum reuse factor per building, respectively \\
\hline$M_{2, o}, M_{28, o}$, and $M_{60, o}$ & Number of RBs of $2 \mathrm{GHz}, 28 \mathrm{GHz}$, and $60 \mathrm{GHz}$ spectra, respectively, of an operator $o$ \\
\hline$P_{2, \mathrm{M}}, P_{2, \mathrm{P}}, P_{28}$, and $P_{60}$ & $\begin{array}{l}\text { The transmission power of a macrocell, a picocell, transceiver } 1 \text { of each small cell, and transceiver } \\
22 \text { of each small cell, respectively }\end{array}$ \\
\hline$\rho_{t, i, o}$ and $\sigma_{t, i, o}(\cdot)$ & SINR and the corresponding link throughput at $\mathrm{RB}=i$ in $\mathrm{TTI}=t$ for an operator $o$ in bps per $\mathrm{Hz}$ \\
\hline $\begin{array}{l}\sigma_{o}^{\mathrm{NR}-\mathrm{U} A n c h}(\cdot), \gamma_{o}^{\mathrm{NR}-\mathrm{U} A n c h}(\cdot) \text {, and } \\
\kappa_{o}^{\mathrm{NR}-\mathrm{U} \text { Anch }}(\cdot)\end{array}$ & System-level average capacity, SE, and EE, respectively, of operator $o$ \\
\hline
\end{tabular}

employed to LTE-LAA standard formalized by 3GPP Release 13 to meet any regional regulatory requirement.

Since in regions where LBT is not mandatory, cellular networks such as LTE-U do not need to have sensing schemes like CSMA/CA in WiFi [21], WiFi may suffer from accessing the shared channel. Hence, to avoid this issue, a separate mechanism is required. In this regard, to address this issue of a cellular network enabled with no LBT feature, the existence of a cellular node with a WiFi AP can be made in the time domain by periodically turning on and off the transmission of the cellular node to allow the WiFi AP to transmit during the off periods. Note that because the exchange of messages for the crosscoordination between different systems is difficult due to the disparity in their protocol development processes described above and vendor differences [28], a noncollaborative coexistence mechanism is suitable for a cellular node and a WiFi AP.

Carrier Sense Adaptive Transmission (CSAT) [29] and FBS allocation are two representative techniques to provide time-domain coexistence $[30,31]$. In CSAT, time is divided into cycles also called CSAT cycles where each cycle consists of on and off periods. Cellular nodes operate during the on period, whereas WiFi APs operate during the off period. Depending on the channel utilization of WiFi APs, the ratio of on to off periods is adjusted to secure fair coexistence. Frequent small gaps (also called punctured subframes) are provided with the on period so that WiFi AP can access these punctured subframes to transmit its delay-sensitive or critical control packets.

Likewise, the ABS-based eICIC technique is an effective procedure to allow the fair coexistence of a cellular node with a WiFi AP [32]. ABSs are low-power subframes that allow only the transmission of the control and reference signals of a cellular node. During ABSs, WiFi APs can detect the vacancy of a channel to transmit data by WiFi APs using the contention-based CSMA/CA protocol. Note that instead of ABSs, FBSs can be used in the unlicensed bands since there is no backward compatibility constraint with previous releases. This is because unlicensed bands are new and do not have to have backward compatibility issues to be maintained with previous generations so that at least control and reference signals are needed to be transmitted at low power during ABSs.

Hence, an FBS is an ABS without transmitting control and reference signals such that it is also termed as absolutely a silent subframe. Note that allocating more subframes to WiFi AP will eventually increase the throughput of the cellular node and vice versa. Moreover, the allocation of FBSs in a time frame can be periodic and aperiodic (or random) [28], and the fairness in resource allocation can be ensured by adopting the number of subframes allocated for WiFi APs in each radio frame [7]. Like CSAT, an FBS also provides divisions in air time to share by a cellular node and $\mathrm{WiFi}$ AP resulting in no transmission of cellular nodes is permitted so that WiFi APs can be allowed to transmit. Based on the local traffic of WiFi APs' traffic load, the number of FBSs can be set. Moreover, FBSs can offer more flexibility than CSAT as the number of subframes can be made adaptive dynamically at the frame level and the position of FBSs in a frame could be noncontiguous.

Hence, in short, the NR-U needs either LBT or FBSbased eICIC technique to protect WiGig from blocking by it. In this paper, we consider investigating the later scenario, i.e., NR-U operating in a region where LBT is not mandatory to employ a cellular node to coexist with a WiGig AP. More specifically, we consider noncollaborative coexistence to apply between NR-U and WiGig by using the FBS-based eICIC such that WiGig can detect and access the vacant channel during FBSs and transmits using its CSMA/CA protocol, which we present in what follows. 


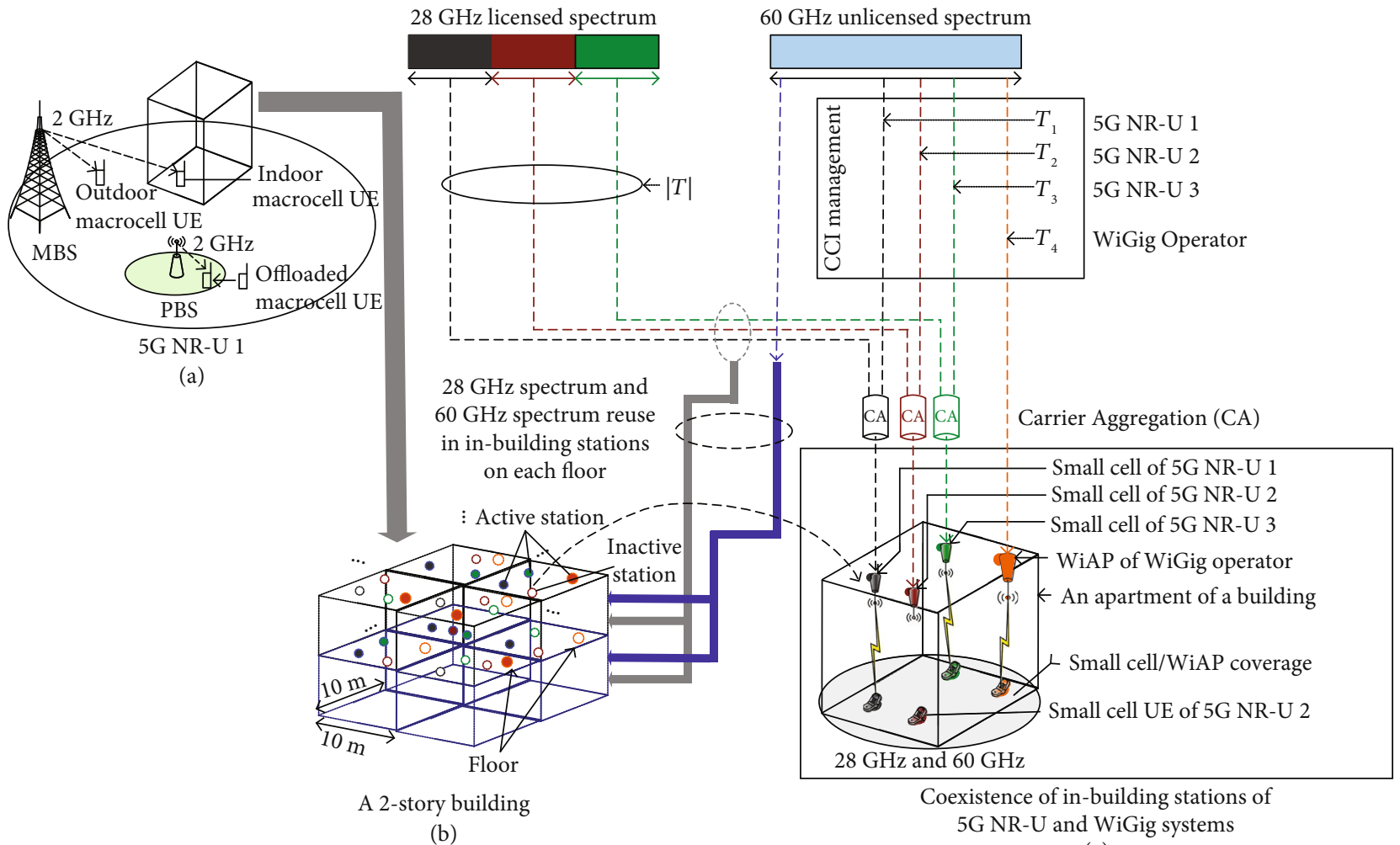

(c)

FIgURE 1: (a) The system architecture of 5G NR-U 1. (b) A 2-story building of small cells of all operators. (c) Coexistence three small cells of three $5 \mathrm{G}$ NR-U operators and a WiAP of one WiGig operator.

\section{System Architecture, Coexistence Scheme, and Interference Management}

3.1. System Architecture. Assume that an arbitrary number of $O_{\mathrm{M}} 5 \mathrm{G}$ NR-U operators and $\mathrm{O}_{\mathrm{W}}$ WiGig operators are in service in a country. Each NR-U operator has three types of base stations (BSs), namely, macrocell BSs (MBSs), picocell BSs (PBSs), and small cell BSs (SBSs). We consider a similar architecture for each NR-U operator such that only one NR-U operator (e.g., NR-U operator 1) is detailed as shown in Figure 1(a). Macrocell user equipments (UEs) can be located indoors and offloaded by outdoor PBSs. Other than the offloaded macrocell UEs, macrocell UEs in the indoor, as well as outdoor, areas are served by an MBS. SBSs and WiGig stations are deployed indoors (i.e., only within multistory buildings), one per apartment per operator in a building (Figure 1(b)). Due to the small coverage and low transmission power of an SBS, as well as a WiGig station, we assume that an SBS or a WiGig station of any operator serves only one UE at a time.

As an extension of the LTE-U or LAA introduced in 3PPP Release-13 [17], we assume that each SBS of an NR$\mathrm{U}$ is both licensed and unlicensed bands enabled using the carrier aggregation technologies, and each band uses a separate transceiver for operation (Figure 1(c)). Due to the favorable propagation characteristics and the availability of large spectrum bandwidths, we consider that each SBS operates in high-frequency bands, including the $28 \mathrm{GHz}$ licensed $\mathrm{mmW}$ band using its transceiver 1 and the $60 \mathrm{GHz}$ unli- censed band using its transceiver 2, to cover a small area at high capacity and high data rates indoors as shown in Figure 1(c). However, both MBSs and PBSs of any NR-U operator operate in the $2 \mathrm{GHz}$ band to cover a large area outdoors.

Each 5G NR-U operator is allocated to an equal amount of the $28 \mathrm{GHz}$ licensed spectrum such that no CCI can be generated from the licensed spectrum. However, due to an unlicensed band, all operators, including all 5G NR-U operators and $\mathrm{WiFi}$ operators, can access the $60 \mathrm{GHz}$ spectrum simultaneously (Figure $1(\mathrm{c})$ ), resulting in generating CCI among them. We present a time-domain-based interference avoidance scheme to avoid CCI generated from the $60 \mathrm{GHz}$ unlicensed spectrum among all 5G NR-U operators and WiGig operators in the following section.

3.2. Proposed Coexistence Mechanism. We present a coexistence mechanism to operate $5 \mathrm{G}$ NR operators in the $60 \mathrm{GHz}$ unlicensed spectrum with incumbent WiGig operators as follows: "A small cell of a $5 \mathrm{G}$ NR operator can share the unlicensed $60 \mathrm{GHz}$ spectrum with a WiGig access point (WiAP) of an incumbent WiGig operator in a multistory building subject to avoiding CCI between them. Moreover, due to a high floor penetration loss in $\mathrm{mmW}$ bands, both the $60 \mathrm{GHz}$ unlicensed spectrum and the dedicated $28 \mathrm{GHz}$ licensed spectrum of each $5 \mathrm{G}$ NR-U operator can be reused to its small cells on each floor of a building."

In this regard, small cells of each $5 \mathrm{G}$ NR-U operator are allocated to a dedicated portion of the $28 \mathrm{GHz}$ licensed 


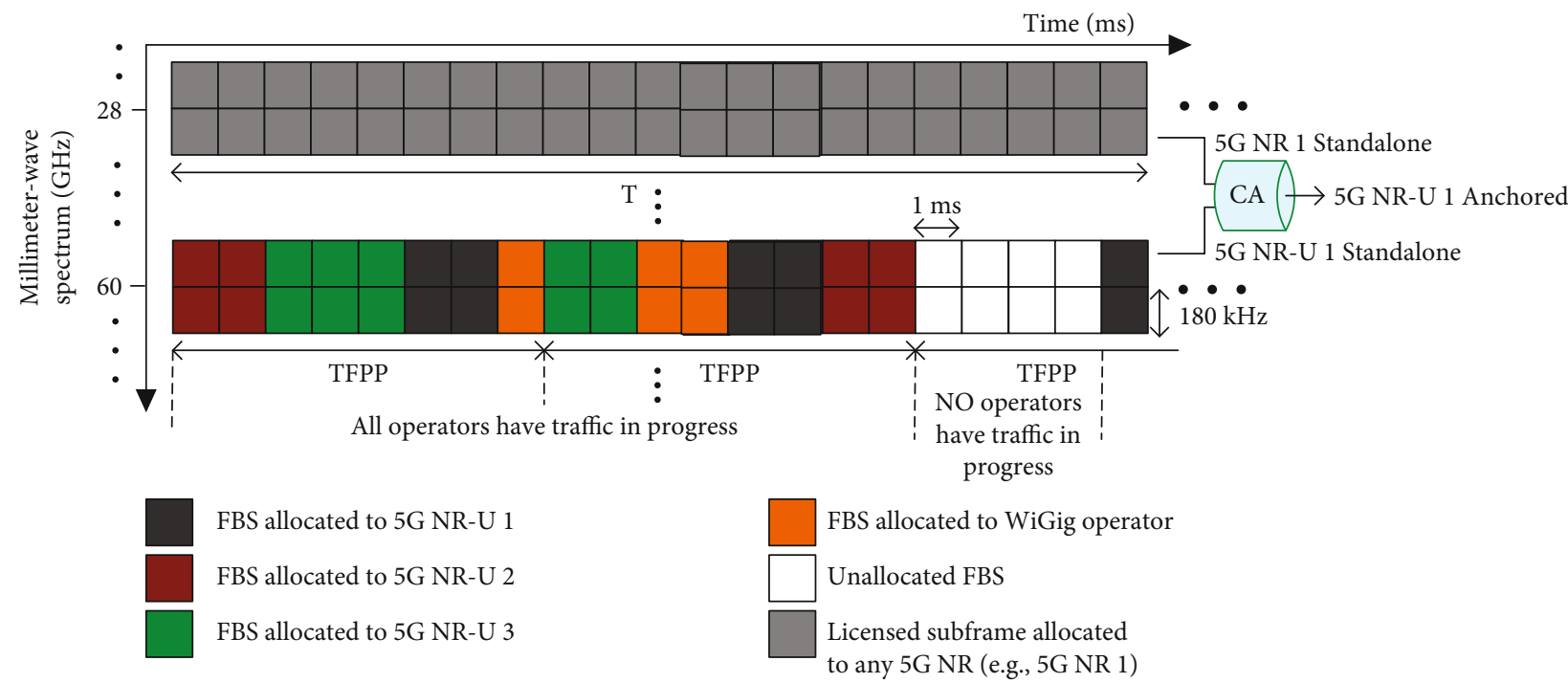

FIGURE 2: CCI interference avoidance mechanism to coexist 5G NR-U and WiGig operators. CA: carrier aggregation.

spectrum by the National Regulatory Agency (NRA) or any third party at the cost of licensing fee. However, due to an unlicensed band, small cells of a 5G NR-U operator can access the whole countrywide $60 \mathrm{GHz}$ spectrum without paying any licensing fees. Further, to avoid CCI of small cells of any 5G NR-U operator in the unlicensed $60 \mathrm{GHz}$ band with WiAPs of any incumbent WiGig operator, a time-domain CCI avoidance scheme following the concept of the ABS-based eICIC technique for the LTE system can be developed, which we describe in the following section. Furthermore, since the floor penetration loss of $\mathrm{mmW}$ signals is high enough [33] to cause insignificant or no CCI among small cells located on adjacent floors, both the $28 \mathrm{GHz}$ licensed and the $60 \mathrm{GHz}$ unlicensed spectra can be reused to small cells of any 5G NR-U operator on each floor of a building, resulting in improving the $\mathrm{mmW}$ spectrum utilization.

3.3. Interference Management. Since each $5 \mathrm{G}$ NR-U operator is allocated to a dedicated portion of the $28 \mathrm{GHz}$ licensed spectrum, no CCI is originated among 5G NR-U operators. However, when operating in the $60 \mathrm{GHz}$ unlicensed spectrum, CCI is originated among all operators, including 5G NR-U operators, as well as WiGig operators, due to accessing the same unlicensed spectrum by all operators. Such CCI in the unlicensed band can be avoided by allocating UEs of each operator orthogonally to the same $60 \mathrm{GHz}$ unlicensed band in the time domain. In time-domain CCI avoidance, UEs of different operators are allocated at a different time such that no two operators can get access to the $60 \mathrm{GHz}$ unlicensed spectrum simultaneously.

One approach to defining an optimal amount of time to transmit data by each operator, in terms of transmission time intervals (TTIs), is to consider the average number of UEs of each operator over a certain duration of time $T$. In this regard, typically, the average number of UEs of one operator differs from the other over $T$ on any floor of a building. This causes the UEs of an operator to get deprived of scheduling sufficient radio resources to them as compared to UEs of other operators. Hence, to overcome the effect of such uneven distribution of UEs within in-building environments, we propose to employ the concept of the ABS-based eICIC technique by modifying ABSs to FBSs, which is defined as subframes with no control signaling, such that an operator $o$ is allowed to access the unlicensed $60 \mathrm{GHz}$ spectrum only during the number of FBSs allocated to it per FBS pattern period (FPP) $T_{\mathrm{FPP}}$ with respect to that of other operators as shown in Figure 2.

Let $O_{\mathrm{M}}$ and $O_{\mathrm{W}}$ denote, respectively, the number of $5 \mathrm{G}$ NR-U operators and WiFi operators in a country. Let $O=($ $\left.O_{\mathrm{M}}+O_{\mathrm{W}}\right)$ denote the total number of operators such that $o \in \mathbf{O}: \mathbf{O}=\{1,2, \cdots, O\}$. We assume that the arrival process of UEs of each operator (both 5G NR-U and WiFi) follows the Poisson process with a mean $\left\{\lambda_{1}, \lambda_{2}, \cdots, \lambda_{O}\right\}$ over $T$. To allow the maximum fairness in the radio resource allocation among operators, we assume that an optimum value of the number of FBSs (i.e., TTIs) $T_{o}$ over $T_{\mathrm{FPP}}$ of any operator $o$ can be obtained in the percentage of $T_{\mathrm{FPP}}$ by taking the ratio of the average number of its UEs to the sum of the average number of UEs of all operators $\lambda_{\mathrm{S}}$ per floor of a building over $T$ as follows:

$$
\begin{aligned}
& T_{o}=\left\lceil\left(\frac{\lambda_{o}}{\lambda_{\mathrm{S}}}\right) T_{\mathrm{FPP}}\right\rceil, \\
& T_{o}=\left\lceil\left(\frac{\lambda_{o}}{\sum_{o=1}^{O} \lambda_{o}}\right) T_{\mathrm{FPP}}\right\rceil .
\end{aligned}
$$

Since the number of FBSs for any operator $o$ over any $T_{\mathrm{FPP}}$ is strictly an integer, $T_{o}$ is leveled to the nearest integer value in the above expression. Note that the value of $T_{o}$ in (1) can be updated depending on the control signaling overhead and the backhaul delay constraints. More specifically, the expression of $T_{o}$ in (1) is suitable for nonideal backhauls with high delay and low control signaling overhead 
requirements, i.e., when the value of $T$ is large enough such that there exists a nonzero value of $\lambda_{o}$ for each operator $o$. However, if $T$ can be small enough (e.g., $T$ is comparable to $\left.T_{\mathrm{FPP}}\right)$ such as for ideal backhauls low delay and high control signaling overheads such that $\lambda_{o}$ can be zero for any operator $o, T_{o}$ can be expressed as follows, which is proved in Proof 1:

$$
T_{o}=\left\lceil\left(\left(\frac{\lambda_{o}}{\sum_{o=1}^{O}\left(1_{v_{o}}\left(\lambda_{o}\right) \times \lambda_{o}\right)}\right) T_{\mathrm{FPP}}\right)\right\rceil .
$$

Proof 1. Let the observation time $T=T_{\mathrm{FPp}}$ since the minimum value of $T$ could be as low as $T_{\text {FPP }}$. Because a UE of small cells of any operator $o$ over any $T_{\text {FPP }}$ may not exist on a floor of a building $l, \lambda_{\mathrm{S}}$ can be expressed as follows:

$$
\lambda_{\mathrm{S}}=\sum_{o=1}^{O}\left(1_{v_{o}}\left(\lambda_{o}\right) \times \lambda_{o}\right)
$$

where $\nu_{o} \in\left\{\lambda_{1}, \lambda_{2}, \lambda_{3}, \cdots, \lambda_{O}\right\} .1(\cdot)$ defines that $1(\cdot)=1$ if $\lambda_{o}$ exists in the set $v_{o}$; otherwise, $1(\cdot)=0$. Now, using (1), we can write the following:

$$
T_{o}=\left\lceil\left(\left(\frac{\lambda_{o}}{\sum_{o=1}^{O}\left(1_{v_{o}}\left(\lambda_{o}\right) \times \lambda_{o}\right)}\right) T_{\mathrm{FPP}}\right)\right\rceil .
$$

\section{Problem Formulation}

4.1. Modeling Traffic Activity of Small Cells. According to [18], sessions or call arrivals can be modeled as a Poisson process. Since given the present state, the future state is independent of the past state, the traffic activity of an inbuilding station (either an SBS or a WiAP) can be modeled as a two-state Markov chain such that the off-state to onstate transition rate of an in-building station is denoted by $\lambda$, whereas the on-state to off-state transition rate is denoted by $\mu$. Let $p(0), p(1), p(2), \cdots, p\left(S_{\mathrm{F}}\right)$ denote onstate probabilities of in-building stations of an operator $o$ on any floor $\mathrm{fl}$, corresponding to its number of active inbuilding stations $s \in\left\{0,1,2, \cdots, S_{\mathrm{F}}\right\}$. The values of these probabilities can be found following the birth-death process. Let $\lambda / \mu=\varepsilon$ such that the probability of $s$ number of active inbuilding stations of an operator $o$ on a floor $\mathrm{fl}$ in a building is given by [18]

$$
p(s)=\frac{S_{\mathrm{F}} !}{s !\left(S_{\mathrm{F}}-s\right) !} \times \varepsilon^{s} \times \frac{1}{(1+\varepsilon)^{S_{\mathrm{F}}}} .
$$

Since an in-building station can serve a maximum of one $\mathrm{UE}$ at a time, the number of stations is equal to the number of in-building UEs that can be served at once for any operator $o$.
Hence, the expected value of the number of in-building UEs of any operator $o$ on a floor $\mathrm{fl}$ within a building is given by

$$
\lambda_{o, s}=E[s]=\sum_{s=0}^{s=S_{\mathrm{F}}}(s \times p(s)) .
$$

4.2. Mathematical Analysis. Assume that the maximum number of WiAPs $W_{\mathrm{AP}, o}$ of each WiGig operator $o$ and the number of SBSs $S_{\mathrm{F}, o}$ of any 5G NR-U operator $o$ are the same on each floor of any building, i.e., $\forall o S_{\mathrm{F}, o}=W_{\mathrm{AP}, o}=S_{\mathrm{F}}$. Let $S_{\mathrm{F}, \text { total }}$ denote the maximum number of small cells of any $5 \mathrm{G} \mathrm{NR}-\mathrm{U}$ operator $o$ per building such that $S_{\mathrm{F} \text {,total }}=\varepsilon_{\mathrm{RF}} \times S_{\mathrm{F}}$ , where $\varepsilon_{\mathrm{RF}}$ is the number of floors per building, defining the vertical spectrum reuse factor per building.

Assume that $L$ denotes the number of buildings per macrocell coverage such that $l \in\{1,2, \cdots, L\}$ of each $5 \mathrm{G}$ NR-U operator. Assume that there are $S_{\mathrm{M}}$ macrocells per $5 \mathrm{G}$ NR-U operator and $S_{\mathrm{P}}$ picocells per macrocell. Let $M_{2,0}$ , $M_{28, o}$, and $M_{60, o}$ denote, respectively, the number of resource blocks (RBs) of $2 \mathrm{GHz}$ microwave spectrum, $28 \mathrm{GHz}$ licensed mmW spectrum, and $60 \mathrm{GHz}$ unlicensed $\mathrm{mmW}$ spectrum of an operator $o$ where an $\mathrm{RB}$ is equal to $180 \mathrm{kHz}$. Note that for all operators, $M_{60, o}$ is the same, i.e., $\forall o M_{60, o}=M_{60}$. Assume also that for all operators, transceiver 1 and transceiver 2 of each small cell operate at the transmission power of $P_{28}$ and $P_{60}$, respectively, whereas the transmission powers of a macrocell and a picocell are denoted as $P_{2, \mathrm{M}}$ and $P_{2, \mathrm{P}}$, respectively.

Let $\mathbf{T}$ denote simulation run time with the maximum time of $Q$ (in time step each lasting $1 \mathrm{~ms}$ ) such that $\mathbf{T}=$ $\{1,2,3, \cdots, Q\}$, and hence, $|\mathbf{T}|=Q$. Let $\mathbf{T}_{o}$ denote the number of FBSs of operator $o$ over $\boldsymbol{T}$. Let $t_{o}$ denote an FBS of operator $o$ such that $t_{o} \in \mathbf{T}_{o}$. Using Shannon's capacity formula, a link throughput at $\mathrm{RB}=i$ in TTI $=t$ for an operator $o$ in bps per $\mathrm{Hz}$ is given by [34-37]

$\sigma_{t, i, o}\left(\rho_{t, i, o}\right)=\left\{\begin{array}{cc}0, & \rho_{t, i, o}<-10 \mathrm{~dB} \\ \beta \log _{2}\left(1+10^{\left(\rho_{t, i, o}(\mathrm{~dB}) / 10\right)}\right), & -10 \mathrm{~dB} \leq \rho_{t, i, o} \leq 22 \mathrm{~dB} \\ 4.4, & \rho_{t, i, o}>22 \mathrm{~dB}\end{array}\right\}$,

where $\beta$ denotes the implementation loss factor. Like $4 \mathrm{G}$ LTE, $\beta$ takes into account the modulation and coding schemes and the Hybrid Automatic Repeat Request mechanisms available in 5G NR [9, 36-39].

The total capacity of all macro UEs serving at the $2 \mathrm{GHz}$ microwave spectrum of any operator $o$ can be expressed as

$$
\sigma_{2, o}=\sum_{t=1}^{Q} \sum_{i=1}^{M_{2, o}} \sigma_{t, i, o}\left(\rho_{t, i, o}\right)
$$

where $\sigma$ and $\rho$ are responses over $M_{2, o}$ RBs of all macro UEs in $t \in \mathbf{T}$ for an operator $o$.

Recall that transceiver 1 of an SBS of each operator $o$ operates at the $28 \mathrm{GHz}$ licensed spectrum such that the 
capacity served by transceiver 1 of an SBS of an operator $o$ is given by

$$
\sigma_{28, o, s}^{\operatorname{Tr} 1}=\sum_{t \in \mathbf{T}} \sum_{i=1}^{M_{28, o}} \sigma_{t, i, o}\left(\rho_{t, i, o}\right) .
$$

Note that the same $28 \mathrm{GHz}$ spectrum is reused to small cells per floor. If all SBSs in each multistory building serve simultaneously in $t \in \mathbf{T}$, then, the aggregate capacity served by transceiver 1 of all SBSs per floor, as well as per building, of operator $o$ is given, respectively, by

$$
\begin{aligned}
\sigma_{28, o, f l}^{\operatorname{Tr} 1} & =\sum_{s=1}^{S_{\mathrm{F}}} \sigma_{28, o, s}^{\operatorname{Tr} 1}, \\
\sigma_{28, o, l}^{\operatorname{Tr} 1} & =\varepsilon_{\mathrm{RF}} \times \sigma_{28, o, f l}^{\operatorname{Tr} 1} .
\end{aligned}
$$

Now, transceiver 2 of all SBSs of operator $o$ per building operates at the $60 \mathrm{GHz}$ spectrum in $t_{o} \in \mathbf{T}_{o}$ such that the capacity served by transceiver 2 of an SBS of an operator $o$ is given by

$$
\sigma_{60, o, s}^{\operatorname{Tr} 2}=\sum_{t_{o} \in \mathbf{T}_{o}} \sum_{i=1}^{M_{60}} \sigma_{t, i, o}\left(\rho_{t, i, o}\right) .
$$

If all SBSs of operator $o$ in each multistory building serve simultaneously in $t_{o} \in \mathbf{T}_{o}$, the aggregate capacity served by transceiver 2 of all SBSs per floor, as well as per building, is given, respectively, by

$$
\begin{aligned}
\sigma_{60, o, f l}^{\operatorname{Tr} 2} & =\sum_{s=1}^{S_{\mathrm{F}}} \sigma_{60, o, s}^{\operatorname{Tr} 2}, \\
\sigma_{60, o, l}^{\operatorname{Tr} 2} & =\varepsilon_{\mathrm{RF}} \times \sigma_{60, o, f l}^{\operatorname{Tr} 2} .
\end{aligned}
$$

Then, the total aggregate capacity served by transceiver 1 and transceiver 2 of all dual-band SBSs per building of operator $o$ is given by

$$
\begin{aligned}
& \sigma_{\mathrm{MB}, o, l}=\sigma_{28, o, l}^{\operatorname{Tr} 1}+\sigma_{60, o, l}^{\operatorname{Tr} 2}, \\
& \sigma_{\mathrm{MB}, o, l}=\varepsilon_{\mathrm{RF}} \times\left(\sigma_{28, o, f l}^{\operatorname{Tr} 1}+\sigma_{60, o, f l}^{\operatorname{Tr} 2}\right) .
\end{aligned}
$$

4.2.1. 5G NR-U Anchored. Due to a short distance between a small cell UE and its SBS and a low transmission power of an SBS, we assume similar indoor signal propagation characteristics for both mmWs for all $L$ buildings per macrocell of an operator $o$. Then, by linear approximation, the system-level average aggregate capacity of operator $o$ for $l>1$ is given by

$$
\begin{aligned}
& \sigma_{o}^{\mathrm{NR}-\mathrm{U} A n c h}(L)=\sigma_{2, o}+\left(L \times \sigma_{\mathrm{MB}, o, l}\right), \\
& \sigma_{o}^{\mathrm{NR}-\mathrm{U} A n c h}(L)=\sigma_{2, o}+\left(L \times \varepsilon_{\mathrm{RF}} \times\left(\sigma_{28, o, f l}^{\mathrm{Tr} 1}+\sigma_{60, o, f l}^{\mathrm{Tr} 2}\right)\right) .
\end{aligned}
$$

The SE for $L$ buildings is then given by

$$
\gamma_{o}^{\mathrm{NR}-\mathrm{U} A n c h}(L)=\frac{\sigma_{o}^{\mathrm{NR}-\mathrm{U} A n c h}(L)}{\left(\left(M_{2, o}+M_{28, o}\right) \times Q\right)} .
$$

Similarly, the EE for $L$ buildings is given by

$$
\kappa_{o}^{\mathrm{NR}-\mathrm{U} A n c h}(L)=\frac{\left(\left(L \times S_{\mathrm{F}} \times\left(P_{28}+P_{60}\right)\right)+\left(S_{\mathrm{P}} \times P_{2, \mathrm{P}}\right)+\left(S_{\mathrm{M}} \times P_{2, \mathrm{M}}\right)\right)}{\left(\sigma_{o}^{\mathrm{NR}-\mathrm{U} A n c h}(L) / Q\right)} .
$$

It is to be noted that for the SE estimation, only the licensed spectra, i.e., $2 \mathrm{GHz}$ and $28 \mathrm{GHz}$ spectra, of each operator $o$ are considered due to paying the licensing fee by the respective operator to use these bands. That is why $60 \mathrm{GHz}$ unlicensed spectrum is not accounted for in the SE estimation because of free of charge to use this band. In other words, only the licensed spectra are considered as the effective spectra of any operator.

4.2.2. 5G NR Standalone and 5G NR-U Standalone. 5G NR standalone and $5 \mathrm{G}$ NR-U standalone operate only in the licensed and unlicensed bands, respectively. The systemlevel average capacity for NR standalone and NR-U standalone can be expressed, respectively, as follows:

$$
\begin{gathered}
\sigma_{o}^{\mathrm{NR} \mathrm{Std}}(L)=\sigma_{2, o}+\left(L \times \varepsilon_{\mathrm{RF}} \times \sigma_{28, o, f l}^{\mathrm{Tr} 1}\right), \\
\sigma_{o}^{\mathrm{NR}-\mathrm{UStd}}(L)=\sigma_{2, o}+\left(L \times \varepsilon_{\mathrm{RF}} \times \sigma_{60, o, f l}^{\mathrm{Tr} 2}\right) .
\end{gathered}
$$

Likewise, following (18) and (19), SE and EE can be expressed using (20) for NR standalone and (21) for NR-U standalone.

\section{Performance Evaluation}

\subsection{Simulation Model}

5.1.1. Default Simulation Parameter and Assumption. Table 4 shows the simulation parameters and assumptions used to evaluate the performance of the proposed scheme. Performance results are generated by a simulator, which is built based on the mathematical analysis given in Section 4, as well as the assumptions, parameters, and models given in Table 4, using the computational tool MATLAB R2012b version running on a personal computer. Default simulation assumptions and parameters used for the performance evaluation are in line with the recommendations from the standardization bodies such as the 3GPP and International Telecommunication Union-Radiocommunication Sector (ITU-R). More specifically, the simulator is developed taking into account the following assumptions and parameters.

Following [40], omnidirectional biconical horn antennas for all small cells and their UEs with a gain of $5 \mathrm{~dB}$ are assumed. Moreover, the proportional fair scheduler to improve the fairness performance in the resource allocation and the full buffer model for the traffic demand in all time over a duration $Q$ are considered for each NR-U operator. 
TABLE 4: Simulation parameters and assumptions.

\begin{tabular}{lc}
\hline Parameters and assumptions & \\
\hline $\begin{array}{l}\text { Maximum number of } \\
\text { operators }\end{array}$ & $5 \mathrm{G}$ NR-U and WiGig \\
& $2 \mathrm{GHz}$ non-LOS \\
Carrier spectrum bandwidth & $28 \mathrm{GHz}$ LOS \\
per 5G NR-U operator & $60 \mathrm{GHz}$ LOS
\end{tabular}

For each $5 G$ NR-U operator

Cellular layout ${ }^{2}$, intersite distance (ISD) ${ }^{1,2}$, transmission direction

Number of cells

Total BS transmit power $(\mathrm{dBm})$

Cochannel small-scale fading model $^{1,5,6}$

MBS and a UE ${ }^{1}$

Path loss

SBS and a $\mathrm{UE}^{1,2,3,5}$

Lognormal shadowing

standard deviation $(\mathrm{dB})$

Antenna configuration

Antenna pattern (horizontal)

Antenna gain plus connector loss ( $\mathrm{dBi})$

UE antenna gain ${ }^{2,3,6}$

$2 \mathrm{GHz}, 28 \mathrm{GHz}$, and $60 \mathrm{GHz}$ (biconical horn)

UE noise figure ${ }^{2,6}$, UE speed ${ }^{1}$, and indoor macrocell UE ${ }^{1}$

Picocell coverage ${ }^{1}$, the total number of macrocell UEs, and macrocell UEs offloaded to all picocells ${ }^{1}$

3D multistory building and SBS models (square-grid apartments)

Number of buildings, number of floors per building Number of apartments per floor, number of SBSs per apartment

Scheduler, traffic model ${ }^{2}$, and type of SBSs

$\mathrm{TTI}^{1}$, FPP, PF scheduler time constant $\left(t_{\mathrm{c}}\right)$, and total simulation run time
Value

3 and 1

$10 \mathrm{MHz}$ (for macrocells and picocells) $50 \mathrm{MHz}$ (for small cells)

$100 \mathrm{MHz}$ (for small cells and WiGig access points)

Hexagonal grid, dense urban, 3 sectors per macrocell site, $1732 \mathrm{~m}$, downlink

$$
\begin{gathered}
1,2 \text {, and } 48 \\
46 \text { and } 37 \\
19 \\
17.3
\end{gathered}
$$

Frequency selective Rayleigh

No small-scale fading effect

No small-scale fading effect

$P L(\mathrm{~dB})=15.3+37.6 \log _{10} R, R$ is in $\mathrm{m}$

$\mathrm{PL}(\mathrm{dB})=15.3+37.6 \log _{10} R+L_{\mathrm{ow}}, R$

is in $\mathrm{m}$ and $L_{\mathrm{ow}}=20 \mathrm{~dB}$

$\mathrm{PL}(\mathrm{dB})=140.7+36.7 \log _{10} R, R$ is in $\mathrm{km}$

$\mathrm{PL}(\mathrm{dB})=61.38+17.97 \log _{10} R, R$ is in $\mathrm{m}$

$\mathrm{PL}(\mathrm{dB})=68+21.7 \log _{10}(R), R$ in $\mathrm{m}$ 8 and 10

9.9 and 0.88

Single-input single-output for all BSs and UEs

Directional $\left(120^{0}\right)$ for $\mathrm{MBS}^{1}$,

omnidirectional for $\mathrm{PBS}^{1}$, and

omnidirectional Biconical horn $\mathrm{SBS}^{1,3}$

$\mathrm{MBS}^{2}, \mathrm{PBS}^{1}$, and $\mathrm{SBS}^{1,3,6} \quad 14,5$, and 5

$0 \mathrm{dBi}, 5 \mathrm{dBi}$, and $5 \mathrm{dBi}$

$9 \mathrm{~dB}$ (for $2 \mathrm{GHz}$ ) and $10 \mathrm{~dB}$ (for

$28 \mathrm{GHz}$ and $60 \mathrm{GHz}$ ), $3 \mathrm{~km} / \mathrm{hr}$, and $35 \%$

$40 \mathrm{~m}$ (radius), 30, 2/15

$$
\begin{gathered}
L, 6 \\
8,1 \\
10 \times 10 \mathrm{~m}^{2}
\end{gathered}
$$

Proportional fair, full buffer, and closed subscriber group femtocell BSs

$1 \mathrm{~ms}, 8 \mathrm{~ms}, 100 \mathrm{~ms}$, and $8 \mathrm{~ms}$

Taken ${ }^{1}$ from [37], ${ }^{2}$ from [43], ${ }^{3}$ from [40], ${ }^{4}$ from [44], from ${ }^{5}$ [45], and from ${ }^{6}$ [46].

Because of the favorable signal propagation, coverage, and hand-off characteristics, we consider the $2 \mathrm{GHz}$ band outdoors, whereas both the $28 \mathrm{GHz}$ and $60 \mathrm{GHz}$ bands indoors. Even though the available bandwidth in the $28 \mathrm{GHz}$ band can be large enough, e.g., $400 \mathrm{MHz}$ [41] or more, for an operator in a country, we consider only $50 \mathrm{MHz}$ per operator for simplicity to evaluate the performance. Likewise, the number of operators in a country can be more than three, and the simulator can be applied to any number of operators in a country.

Due to the less multipath fading effect of high-frequency signals in indoor environments, we consider the Line-OfSight (LOS) large-scale path loss model for the $\mathrm{mmW}$ signals 
within buildings. Similarly, because of a small coverage and less multipath fading effect of an indoor small cell operating in the mmW bands, we assume a similar mmW signal propagation characteristic within each adjacent building. Moreover, due to the high external wall and floor penetration losses of a building, low transmission power of a small cell, and high attenuation experienced by high-frequency signals, both the $28 \mathrm{GHz}$ and $60 \mathrm{GHz}$ spectrum bands can be reused in small cells within the same building, as well as adjacent buildings. Since information is transmitted in every discrete TTI in mobile systems, we consider the discrete-event type simulation at TTI of $1 \mathrm{~ms}$ to execute simulation results. We limit evaluating the performance of only cellular networks (i.e., for $5 \mathrm{G}$ NR-U networks only) in the unlicensed bands for a number of evaluation scenarios. WiGig-related performance evaluations are out of the scope of this paper, and hence, no results and analyses for WiGig networks are presented.

5.1.2. The Operating Mechanism of the Simulator. In the following, we describe in brief how the simulator works. For each operator, the total number of macrocell UEs are first disjointed into three groups to estimate indoor, outdoor, and offloaded (to picocells) macrocell UEs randomly. The realization of macrocell UEs served by the macrocell and picocells is not mutually independent since macrocell UEs served by picocells are macrocell UEs offloaded from the macrocell, and the schedulers have complete knowledge when a macrocell UE is offloaded [42]. The number of small cell buildings and picocells is located randomly and uniformly in the macrocell area of each operator. The indoor macrocell UEs are distributed randomly and nonuniformly within each building. All outdoor macrocell UEs, offloaded macrocell UEs, and small cell UEs are distributed randomly and uniformly within their respective BSs' coverage area.

All macrocell UEs are then scheduled by a frequencydomain proportional fair scheduler at the MBS. Likewise, for each operator, a frequency-domain proportional fair scheduler for all small cells per floor per $\mathrm{mmW}$ band in a building schedules small cell UEs of the respective floor. The same process repeats for all RBs of each spectrum band per operator in each TTI and continues for all operators for all TTIs of the simulation run time. The aggregate throughput is then estimated for all macrocell UEs using (9), whereas for all small cell UEs per operator per building using (15). The aggregate throughput of small cell UEs for all buildings per operator is then estimated and summed with that of macrocell UEs using (17) for NR-U anchored. Likewise, the system-level average SE and EE are estimated using (18) and (19), respectively, for NR-U anchored. The same process repeats for all operators.

Now, following the same procedure, the average capacity for NR standalone and NR-U standalone for all operators can be estimated using (20) and (21), respectively. Similarly, following (18) and (19), SE and EE for NR standalone and NR-U standalone can be estimated. Note that we have explained the calculation of throughput in Section 4.2. Moreover, the shadow fading and small-scale fading of all UEs are estimated and updated in each TTI per realization.
5.2. Performance Evaluation Scenario. We consider three $5 \mathrm{G}$ NR-U operators and one WiGig operator. The following two scenarios are evaluated for $5 \mathrm{G}$ NR-U operators:

(i) A single 5G NR-U operator and a single WiGig operator

(ii) Multiple 5G NR-U operators and a single WiGig operator

In the former scenario, performance metrics are evaluated by varying the transmission time per FPP of a $5 \mathrm{G}$ NR-U operator for $50 \%, 75 \%$, and $100 \%$ of FPP by changing its average number of small cell UEs on each floor of a building. For the latter scenario, three $5 \mathrm{G}$ NR-U operators (i.e., 5 G NR-U operator 1, 5G NR-U operator 2, and 5G NR-U operator 3) are considered without changing the number of WiGig operators to evaluate the SE and EE performances of $5 \mathrm{G}$ NR-U operators for an arbitrary average number of small cell UEs of $1 / 8,2 / 8$, and $3 / 8$ per FPP, respectively.

For the worst-case evaluation, we consider the maximum amount of interference that any NR-U node (i.e., small cell) or the AP of the WiGig operator (i.e., WiAP) may get experienced in the presence of others in each apartment of a building. Hence, theoretically, each 5G NR-U operator has the maximum number of one node, as well as the WiGig operator has the maximum number of one AP, in each apartment of a building. However, in practice, a node of any $5 \mathrm{G}$ NR-U operator and the AP of the WiGig operator may not be deployed in an apartment such that the distribution of the total number of nodes and APs per apartment of a building is random. Hence, the average interference experienced by a node or the AP is upper limited by the amount of interference it experienced in the worst-case scenario. Moreover, a node of any 5G NR-U operator, or the AP of the WiGig operator, could be deployed and run by either the corresponding operator or the customer without any explicit coordination between the 5G NR-U operator and the WiGig operator due to the disparity in the development of their MAC layers' protocols, mentioned earlier.

\subsection{Performance Result and Analysis}

5.3.1. Coexistence of a Single 5G NR-U Operator and a Single WiGig Operator. Figure 3 shows the average capacity, SE, and $\mathrm{EE}$ responses of small cells of a single operator of $5 \mathrm{G}$ NR standalone (Std), 5G NR-U Std, and 5G NR-U anchored (Anch) systems with the variation in its transmission time (i.e., the number of FBSs allocated to it) per FPP, including $50 \%, 75 \%$, and $100 \%$ of FPP, of the respective operator of each system. The operator of each $5 \mathrm{G}$ NR system is considered coexisting with a single WiGig operator within a multistory building for the vertical reuse factor $(\mathrm{vRF})=1$ and horizontal $\mathrm{RF}(\mathrm{hRF})=1$. Note that the SE of an operator is influenced by its effective licensed spectrum, as well as its achievable capacity. However, the achievable capacity is affected mainly by the transmission time over an FPP and the available licensed and unlicensed spectra of an operator. Like SE, EE is influenced by the capacity, as well as the transmission power of SBSs of an operator. 


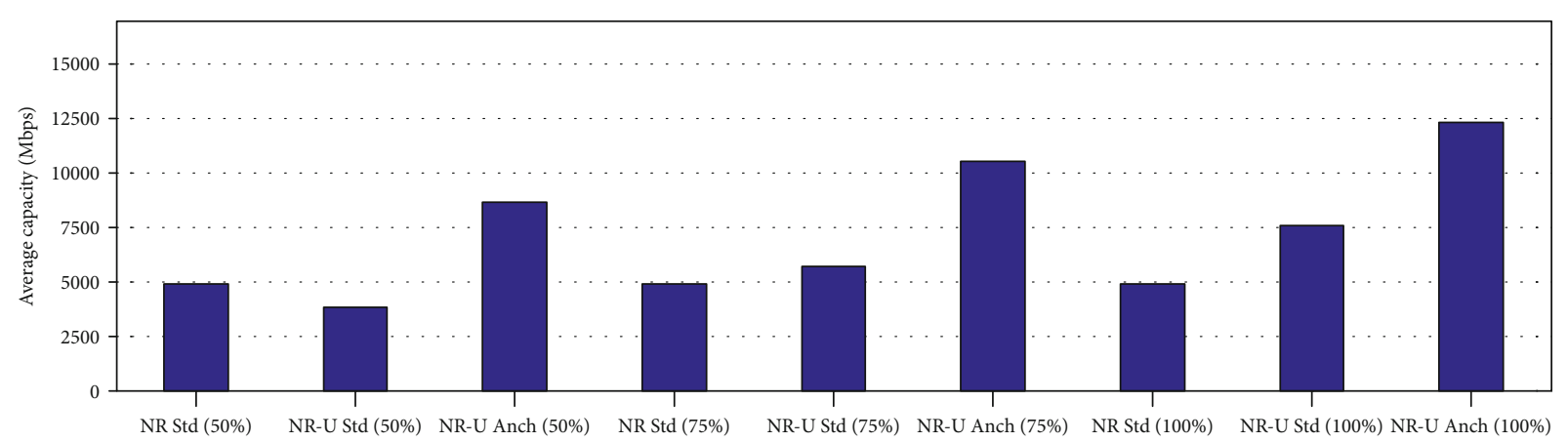

(a)

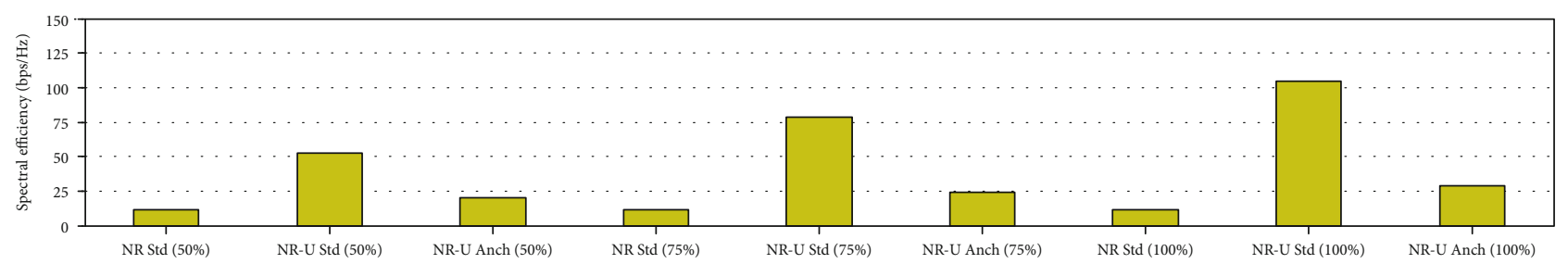

(b)

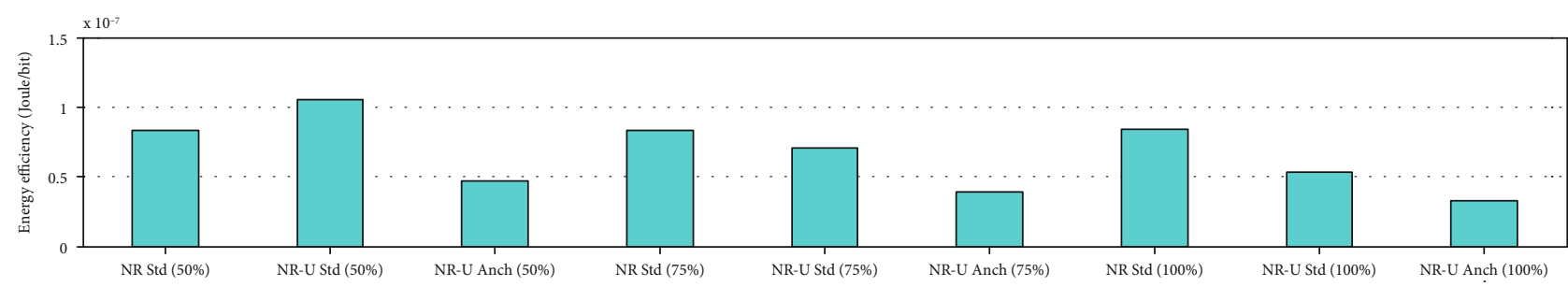

(c)

Figure 3: Average capacity, SE, and EE responses of small cells of a single operator of 5G NR standalone (Std), 5G NR-U Std, and 5G NR-U anchored (Anch) systems with the variation in its transmission time per FPP, including 50\%, 75\%, and $100 \%$ of FPP, which coexists with a single WiGig operator in a building for $\mathrm{vRF}=1$ and $\mathrm{hRF}=1$. (a) average capacity, (b) SE, and (c) EE.

Since the $28 \mathrm{GHz}$ licensed spectrum is allocated exclusively to an NR operator, no changes in capacity, SE, and EE due to the licensed band can occur with a change in the number of FBSs over an FPP. This can be observed for NR standalone since in-building small cells of the NR standalone operator operate only in the $28 \mathrm{GHz}$ licensed spectrum. This causes NR standalone small cells to be independent of the transmission time, the available licensed spectrum, and the overall transmission power of all small cells. However, as small cells of the NR-U are allowed more time to transmit (e.g., increasing the transmission time from $50 \%$ FPP to $100 \%$ FPP) only in the $60 \mathrm{GHz}$ unlicensed spectrum, the capacity, SE, and EE of the NR-U operator are increased.

Further, with an increase in the transmission time, the NR-U anchored operator provides the best responses in average capacity and EE. The average capacity of NR-U anchored operator is increased due to operating in both the $28 \mathrm{GHz}$ licensed and the $60 \mathrm{GHz}$ unlicensed spectra, as compared to only the $28 \mathrm{GHz}$ for the NR standalone and the $60 \mathrm{GHz}$ for the NR-U standalone operators. The increase in capacity is significant enough to exceed the corresponding increase in the transmission energy with a high margin due to increasing the transmission time from 50\% FPP to $100 \%$
FPP such that the required average energy per bit transmission is the least.

Furthermore, though the SE of the NR-U anchored operator increases with an increase in the transmission time, the best SE response is achieved when small cells of the NR$\mathrm{U}$ operator are operating only in the $60 \mathrm{GHz}$ unlicensed spectrum due to requiring the least amount of the effective licensed spectrum by the NR-U operator. Overall, NR-U anchored provides the best capacity and EE responses, whereas NR-U standalone provides the best SE response. Since the unlicensed spectrum is common to both schemes, this implies the importance of operating the 5G NR in the unlicensed bands.

5.3.2. Coexistence of Multiple $5 G$ NR-U Operators and a Single WiGig Operator and the Impact of Spectrum Reuse Factors. Figure 4 shows SE and EE responses when multiple NR-U anchored operators coexist with a single WiGig operator for the vRF of 1 and 6 with the variation of the hRF from 1 to $L=50$. vRF is obtained by reusing both the $28 \mathrm{GHz}$ and $60 \mathrm{GHz} \mathrm{mmW}$ spectra of the NR-U anchored operator to its small cells located on each floor of a sixstory building, for example, whereas hRF is obtained by 


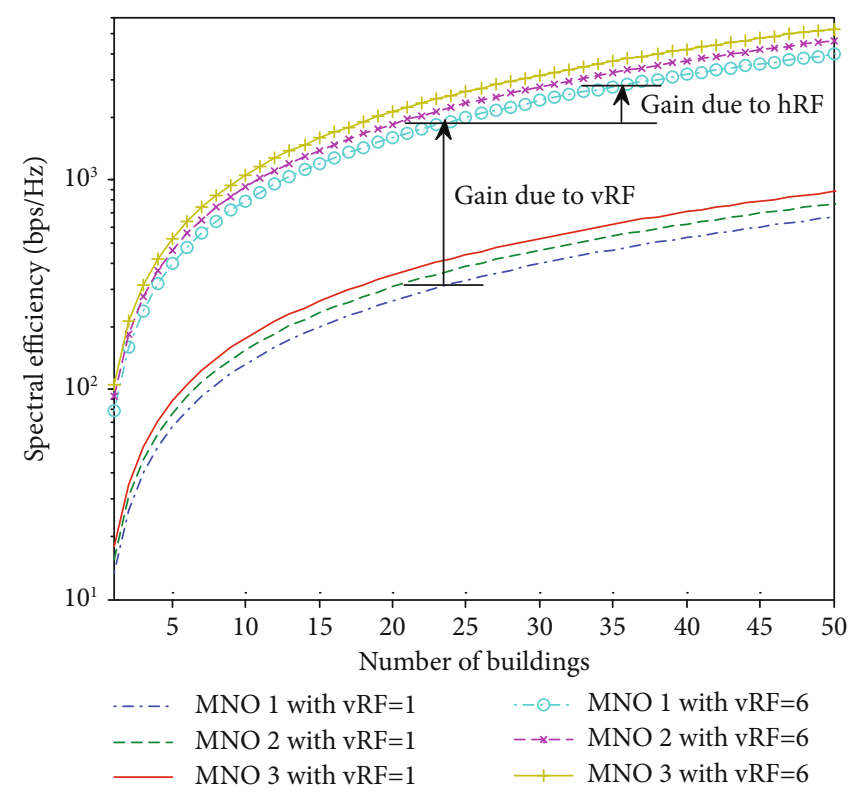

(a)

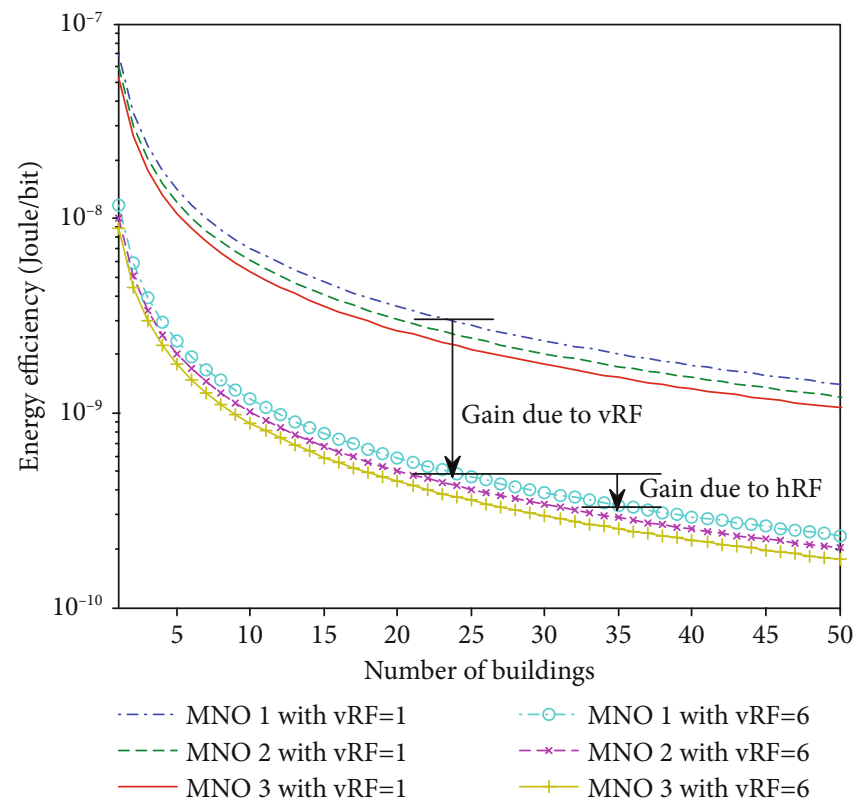

(b)

FIGURE 4: SE and EE responses of three 5G NR-U anchored operators when coexisting with a single WiGig operator by varying the transmission time of $12.5 \% \mathrm{FPP}, 25 \% \mathrm{FPP}, 37.5 \% \mathrm{FPP}$, and 25\%FPP, respectively, per FPP for the vRF of 1 and 6 and the hRF of 1 to $L$ $=50$.

reusing these spectra to $L$ number of six-story buildings over a macrocell coverage. From Figure 4, it can be observed that an NR-U anchored operator (i.e., NR-U operator 3) allocated to more FBSs per FPP can provide better SE and EE performances than others (i.e., NR-U operator 1 and NR-U operator 2). Also, due to allocating less number of FBSs per FPP to an NR-U anchored operator, SE and EE per NR-U operators decrease with an increase in the number of NR-Us. The above explanation is applicable for multiple WiGig operators coexisting with other NR-U schemes.

5.4. Performance Comparison. Assume that the future $6 \mathrm{G}$ systems would require 10 times average SE (i.e., 270$370 \mathrm{bps} / \mathrm{Hz}$ ) and 10 to 100 times average EE (i.e., $0.3 \times$ $10^{-6}$ to $0.03 \times 10^{-6}$ Joules/bit) of $5 \mathrm{G}$ systems [47-50]. Considering $370 \mathrm{bps} / \mathrm{Hz}$ SE and $0.03 \mu \mathrm{J} / \mathrm{bit} \mathrm{EE}$ requirements for $6 \mathrm{G}$, from Figure 3, it can be found that without reusing the $\mathrm{mmW}$ spectra spatially, it is difficult to satisfy these requirements for $6 \mathrm{G}$ with any $5 \mathrm{G} \mathrm{NR}$ schemes. However, from Figure 4 , it can be found that by choosing an appropriate value of vRF, as well as hRF, the expected SE and EE requirements for $6 \mathrm{G}$ mobile systems mentioned above can be satisfied. For example, considering the worst-case analysis, NR-U anchored (5G NR-U operator 1) with the minimum number of FBSs for the transmission per FPP can achieve both the expected $\mathrm{SE}$ and $\mathrm{EE}$ requirements for $6 \mathrm{G}$ for either $\mathrm{vRF}=1$ and $\mathrm{hRF}=28$ or $\mathrm{vRF}=6$ and $\mathrm{hRF}=5$.

\section{Conclusion and Future Research Direction}

The cost and scarcity of the available licensed spectrum are major concerns toward addressing the continuing demand for high capacity and data rate at a low cost per bit transmis- sion in cellular networks. Due to the license-free access to unlicensed bands and wide spectrum availability in both unlicensed and licensed bands, the operation in $\mathrm{mmW}$ bands is considered as a potential solution to minimize spectrum scarcity and licensing cost for $5 \mathrm{G}$ NR and beyond mobile networks. In this regard, due to wider contiguous bandwidth availability, the $60 \mathrm{GHz}$ unlicensed $\mathrm{mmW}$ band is considered an attractive candidate to operate $5 \mathrm{G}$ NR-U. However, the IEEE 802.11-based WiGig is in operation in the $60 \mathrm{GHz}$ band. Hence, to operate in the $60 \mathrm{GHz}$ unlicensed band, an appropriate mechanism is necessary for a $5 \mathrm{G}$ NR-U operator to coexist with an incumbent WiGig operator without causing interference to each other.

Accordingly, in this paper, we have given an overview, including issues, challenges, and possible solution alternatives, for the fair coexistence of cellular and incumbent WiFi networks in the unlicensed bands. We then have presented a coexistence mechanism for 5G NR-U small cells located within buildings to coexist with WiGig operators. Each small cell is dual-band enabled operating in the $60 \mathrm{GHz}$ unlicensed and $28 \mathrm{GHz}$ licensed $\mathrm{mmW}$ bands. Following several research studies that proposed to use the ABS-based eICIC technique in LTE to address the coexistence issue between WiFi and cellular operators in the unlicensed band, we have developed an interference avoidance scheme that considers FBSs by modifying the concept of ABS to avoid CCI between small cells of the 5G NR-U operator and the incumbent WiGig operator. We have then derived average capacity, $\mathrm{SE}$, and EE responses of in-building 5G NR-U small cells. The system-level performance analysis has been carried out for a number of variants of $5 \mathrm{G}$ NR-U, including $5 \mathrm{G} \mathrm{NR}$ standalone, 5G NR-U standalone, and 5G NR-U anchored. In addition, we have analyzed the impact of reusing $\mathrm{mmW}$ spectra by varying 
both the vertical and horizontal spatial spectrum reuse factors, as well as the coexistence of multiple 5G NR-U operators with a WiGig operator.

It has been shown that $5 \mathrm{G} \mathrm{NR}-\mathrm{U}$ anchored provides the best capacity and EE performances, whereas $5 \mathrm{G}$ NR-U standalone provides the best SE performances, which implies explicitly the importance of operating the 5G NR on unlicensed bands as the $60 \mathrm{GHz}$ unlicensed spectrum is common to both schemes. For the coexistence of multiple $5 \mathrm{G}$ NR-U anchored operators, it has been found that any $5 \mathrm{G}$ NR-U allocated to more FBSs per FPP can provide better SE and EE performances than others. Moreover, due to allocating less number of FBSs to serve traffic per FPP to a $5 \mathrm{G}$ NR-U, the SE and EE per 5G NR-U decrease with an increase in the number of $5 \mathrm{G}$ NR-U operators. For the impact of spatial spectrum reuse factors, it has been shown that regardless of the number of $5 \mathrm{G}$ NR-U operators, an increase in either vertical reuse factor (vRF), or horizontal reuse factor (hRF), both SE and EE improve. Moreover, by choosing an appropriate value of $\mathrm{vRF}$, as well as hRF, the expected SE and EE requirements for $6 \mathrm{G}$ mobile systems can be achieved.

In this paper, we limit evaluating the proposed coexistence mechanism to the performance of cellular technologies, i.e., NR-U under different deployment scenarios, in the presence of IEEE 802.11 technologies, i.e., WiGig, in the high-frequency $60 \mathrm{GHz} \mathrm{mmW}$ band. In our future studies, we will be interested in evaluating the performance of $\mathrm{WiGig}$ technologies as well to see the combined effect of the proposed mechanism on both technologies. Since the performance of the proposed mechanism depends on how we allocate blank subframes to cellular and IEEE 802.11 standards, other approaches than the one used in this paper (i.e., the average number of UEs per operator over a certain duration $T$ ) to define the optimal number of blank subframes to transmit data by each operator can be explored to improve the combined performance of all operators further.

Further, even though the impact of the assumption about serving multiple UEs simultaneously by each SBS/WiGig AP is insignificant over $T$ in terms of the throughput performance, which may as well lead to the increased complexity in analysis and the difficulty in deriving closedform expressions, it would be interesting to see the overall performance when investigating under such multiple UEs per SBS/WiGig AP scenario. Besides, though the blank subframe mechanism reduces delay in cellular users due to a small gap between transmissions, a short sensing period may lead to inaccurate channel sensing results. Hence, a tradeoff between interference detection accuracy and cellular user experience is another important research direction to carry out. Few other directions for extension may include investigating the proposed mechanism under other unlicensed spectrum bands, particularly, $6 \mathrm{GHz}$, beam-based transmissions, and distributed time resource allocations.

\section{Data Availability}

Data, primarily, in the form of numerous simulation assumptions and parameters reported previously by the standardization bodies, including $3^{\text {rd }}$ Generation Partnership Project
(3GPP) [42, 43] and International-Telecommunication Union-Radiocommunication Sector (ITU-R) [46], included and detailed within the article in Table 4, were used to carry out the performance evaluation of this study. Other prior studies than these above were cited as references [39, 44, 45] as well. No data other than these were used to evaluate the performance studies. Taking into account all these parameters and assumptions, performance results were generated by a simulator running on a personal computer, which was built by the author using the standard computational tool MATLAB R2012b. MATLAB codes are not publicly available. However, supports for writing MATLAB instruction codes can be provided over the emails querying directly to the author at rony107976@gmail.com.

\section{Conflicts of Interest}

The author declares that there is no conflict of interest regarding the publication of this paper.

\section{References}

[1] A. Bleicher, "A surge in small cells [2013 Tech To Watch]," IEEE Spectrum, vol. 50, no. 1, pp. 38-39, 2013.

[2] Y. Jiang, J. Guo, and Z. Fei, "Performance analysis of the coexistence of $5 \mathrm{G}$ NR-unlicensed and Wi-Fi with mode selection," in 2020 IEEE/CIC International Conference on Communications in China (ICCC), pp. 953-958, Chongqing, China, 2020.

[3] R. Zhang, M. Wang, L. X. Cai, Z. Zheng, X. Shen, and L. Xie, "LTE-unlicensed: the future of spectrum aggregation for cellular networks," IEEE Wireless Communications, vol. 22, no. 3, pp. 150-159, 2015.

[4] S. Lagen, N. Patriciello, and L. Giupponi, "Cellular and Wi-Fi in unlicensed spectrum: competition leading to convergence," in 2020 2nd 6 G Wireless Summit (6G SUMMIT), pp. 1-5, Levi, Finland, 2020.

[5] A. M. Baswade, M. Reddy, A. Antony Franklin, B. R. Tamma, and V. Sathya, "Performance analysis of spatially distributed LTE-U/NR-U and Wi-Fi networks: an analytical model for coexistence study," Journal of Network and Computer Applications, vol. 191, 2021.

[6] T. Nihtila, V. Tykhomyrov, O. Alanen et al., "System performance of LTE and IEEE 802.11 coexisting on a shared frequency band," in 2013 IEEE Wireless Communications and Networking Conference (WCNC), pp. 1038-1043, Shanghai, China, 2013.

[7] Y. Huang, Y. Chen, Y. T. Hou, W. Lou, and J. H. Reed, "Recent advances of LTE/WiFi coexistence in unlicensed spectrum," IEEE Network, vol. 32, no. 2, pp. 107-113, 2018.

[8] G. Naik, J.-M. Park, J. Ashdown, and W. Lehr, "Next generation Wi-Fi and 5G NR-U in the $6 \mathrm{GHz}$ bands: opportunities and challenges," IEEE Access, vol. 8, pp. 153027-153056, 2020.

[9] S. Lagen, L. Giupponi, S. Goyal et al., "New radio beam-based access to unlicensed spectrum: design challenges and solutions," IEEE Communications Surveys \& Tutorials, vol. 22, no. 1, pp. 8-37, 2020.

[10] N. Patriciello, S. Goyal, S. Lagen et al., "NR-U and WiGig coexistence in $60 \mathrm{GHz}$ bands," 2020, http://arxiv.org/abs/2001 .04779 .

[11] N. Patriciello, S. Lagen, B. Bojovic, and L. Giupponi, "NR-U and IEEE 802.11 technologies coexistence in unlicensed 
mmWave spectrum: models and evaluation," IEEE Access, vol. 8, pp. 71254-71271, 2020.

[12] J. Verboom and S. Kim, "Stochastic analysis on downlink performance of coexistence between WiGig and NR-U in $60 \mathrm{GHz}$ band," 2020, http://arxiv.org/abs/2003.01570.

[13] E. Almeida, A. M. Cavalcante, R. C. D. Paiva et al., "Enabling LTE/WiFi coexistence by LTE blank subframe allocation," in 2013 IEEE International Conference on Communications (ICC), pp. 5083-5088, Budapest, 2013.

[14] S. Chatterjee, M. J. Abdel-Rahman, and A. B. MacKenzie, "Optimal distributed allocation of almost blank subframes for LTE/WiFi coexistence," in 2017 15th International Symposium on Modeling and Optimization in Mobile, Ad Hoc, and Wireless Networks (WiOpt), pp. 1-7, Paris, France, 2017.

[15] A. M. Cavalcante, E. Almeida, R. D. Vieira et al., "Performance Evaluation of LTE and Wi-Fi Coexistence in Unlicensed Bands," in 2013 IEEE 77th Vehicular Technology Conference (VTC Spring), pp. 1-6, Dresden, Germany, 2013.

[16] A. Al-Dulaimi, S. Al-Rubaye, Q. Ni, and E. Sousa, "5G communications race: pursuit of more capacity triggers LTE in unlicensed band," IEEE Vehicular Technology Magazine, vol. 10, no. 1, pp. 43-51, 2015.

[17] X. Lu, M. Lema, T. Mahmoodi, and M. Dohler, "Downlink data rate analysis of $5 \mathrm{G}-\mathrm{U}$ ( $5 \mathrm{G}$ on unlicensed band): coexistence for 3GPP 5G and IEEE802.11ad WiGig," in 23th European Wireless Conference, pp. 1-6, Dresden, Germany, 2017.

[18] Q. Cui, Y. Gu, W. Ni, and R. P. Liu, "Effective capacity of licensed-assisted access in unlicensed spectrum for 5G: from theory to application," IEEE Journal on Selected Areas in Communications, vol. 35, no. 8, pp. 1754-1767, 2017.

[19] E. Puschita and T. Palade, "Performance analysis of contention-based channel access mechanisms of 802.11 DCF and 802.11e EDCA," in 2009 9th International Conference on Telecommunication in Modern Satellite, Cable, and Broadcasting Services, pp. 548-551, 2019.

[20] "The 3rd generation partnership project," http://www.3gpp .org.

[21] M. Alhulayil and M. Lopez-Benitez, "Coexistence mechanisms for LTE and Wi-Fi networks over unlicensed frequency bands," in 2018 11th International Symposium on Communication Systems, Networks \& Digital Signal Processing (CSNDSP), pp. 1-6, Budapest, Hungary, 2018.

[22] R. Ratasuk, N. Mangalvedhe, and A. Ghosh, "LTE in unlicensed spectrum using licensed-assisted access," in 2014 IEEE Globecom Workshops (GC Wkshps), pp. 746-751, Austin, TX, USA, 2014.

[23] R. Kwan, R. Pazhyannur, J. Seymour et al., "Fair co-existence of Licensed Assisted Access LTE (LAA-LTE) and Wi-Fi in unlicensed spectrum," in 2015 7th Computer Science and Electronic Engineering Conference (CEEC), pp. 13-18, Colchester, UK, 2015.

[24] 3GPP, "Study on NR-based access to unlicensed spectrum," in document TSG RAN 75 Meeting, RP-170828, 3GPP, Sophia Antipolis, France, 2017.

[25] F. S. Chaves, E. P. L. Almeida, R. D. Vieira et al., "LTE UL power control for the improvement of LTE/Wi-Fi coexistence," in 2013 IEEE 78th Vehicular Technology Conference (VTC Fall), pp. 1-6, Las Vegas, NV, USA, 2013.

[26] Qualcomm Technologies, "LTE in unlicensed spectrum: harmonious coexistence with WiFi," 2014, http://www.qualcomm .com/media/documents/files/lte-unlicensedcoexistencewhitepaper.pdf.

[27] R. K. Saha, "Coexistence of cellular and IEEE 802.11 technologies in unlicensed spectrum bands -a survey," IEEE Open Journal of the Communications Society, vol. 2, pp. 1996-2028, 2021.

[28] H. Zhang, X. Chu, W. Guo, and S. Wang, "Coexistence of Wi$\mathrm{Fi}$ and heterogeneous small cell networks sharing unlicensed spectrum," IEEE Communications Magazine, vol. 53, no. 3, pp. 158-164, 2015.

[29] LTE in Unlicensed Spectrum: Harmonious Coexistence with Wi-Fi, Qualcomm Technologies, Inc., San Diego, CA, USA, 2014, https://www.qualcomm.com/media/documents/files/ lte-unlicensed-coexistence-whitepaper.pdf.

[30] Z. Guan and T. Melodia, "CU-LTE: spectrally-efficient and fair coexistence between LTE and Wi-Fi in unlicensed bands," in IEEE INFOCOM 2016- The 35th Annual IEEE International Conference on Computer Communications, pp. 1-9, San Francisco, CA, USA, 2016.

[31] R. K. Saha, "On operating cellular technologies in unlicensed spectrum bands: A review," in The Sixteenth International Conference on Systems and Networks Communications (ICSNC 2021), Barcelona, Spain, 2021.

[32] R. K. Saha, "Spectrum allocation and reuse in 5G new radio on licensed and unlicensed millimeter-wave bands in indoor environments," Mobile Information Systems, vol. 2021, Article ID 5538820, 21 pages, 2021.

[33] R. K. Saha, "3D spatial reuse of multi-millimeter-wave spectra by ultra-dense in-building small cells for spectral and energy efficiencies of future $6 \mathrm{~g}$ mobile networks," Energies, vol. 13, no. 7, 2020.

[34] 3GPP; Technical specification Group Radio Access Network, Evolved Universal Terrestrial Radio Access (E-UTRA); FDD Home eNode B (HeNB) Radio Frequency (RF) Requirements Analysis (Release 9), 3rd Generation Partnership Project, 2010, TR 36.921, 9.0.0.

[35] J. Ellenbeck, J. Schmidt, U. Korger, and C. Hartmann, “A concept for efficient system-level simulations of OFDMA systems with proportional fair fast scheduling," in IEEE GLOBECOM Workshops, pp. 1-6, Honolulu, HI, USA, 2009.

[36] R. K. Saha and P. Saengudomlert, "Novel resource scheduling for spectral efficiency in LTE-advanced systems with macrocells and femtocells," in The 8th Electrical Engineering/Electronics, Computer, Telecommunications and Information Technology (ECTI) Association of Thailand-Conference 2011, pp. 340-343, Khon Kaen, Thailand, 2011.

[37] "Evolved universal terrestrial radio access (E-UTRA); radio frequency (RF) system scenarios," in Document 3GPP TR 36.942, V.1.2.0, 3rd Generation Partnership Project, 2007, https://portal.3gpp.org/desktopmodules/Specifications/ SpecificationDetails.aspx? specificationId $=2592$.

[38] Techplayon, "5G NR modulation and coding schememodulation and code rate," July 2021, https://www.techplayon .com $/ 5 \mathrm{~g}$-nr-modulation-and-coding-scheme-modulation-andcode-rate/.

[39] 3GPP, "Discussion on HARQ enhancements for NR-U document," in 3GPP TSG RAN WG1 93 Meeting R1-1806671 NEC, Tokyo Japan, 2018.

[40] S. Geng, J. Kivinen, X. Zhao, and P. Vainikainen, "Millimeterwave propagation channel characterization for short-range wireless communications," IEEE Transactions on Vehicular Technology, vol. 58, no. 1, pp. 3-13, 2009. 
[41] Japan Assigns 5G Spectrum to Four Operators-5G Observatory, Publications Office of the European Union, Luxembourg, Europe, 2021, https://5gobservatory.eu/japan-assigns-5gspectrum-to-four-operators/.

[42] R. K. Saha and C. Aswakul, "A novel frequency reuse technique for in-building small cells in dense heterogeneous networks," IEEJ Transactions on Electrical and Electronic Engineering, vol. 13, no. 1, pp. 98-111, 2018.

[43] 3GPP, "Simulation assumptions and parameters for FDD HeNB RF requirements," in document TSG RAN WG4 (Radio) Meeting \#51, R4-092042, 3GPP, San Francisco, USA, May 2009https://www.3gpp.org/ftp/tsg_ran/WG4_Radio/TSGR4_ 51/Documents/.

[44] R. K. Saha, P. Saengudomlert, and C. Aswakul, "Evolution toward $5 \mathrm{G}$ mobile networks-a survey on enabling technologies," Engineering Journal, vol. 20, no. 1, pp. 87-119, 2016.

[45] G. R. Maccartney, T. S. Rappaport, S. Sun, and S. Deng, "Indoor office wideband millimeter-wave propagation measurements and channel models at 28 and $73 \mathrm{GHz}$ for ultradense 5G wireless networks," IEEE Access, vol. 3, pp. 23882424, 2015.

[46] Study Group (ITU-R), "Guidelines for evaluation of radio interface technologies for IMT-2020," Report ITU-R M.24120 (10/2017), Geneva, 2017https://www.itu.int/dms_pub/itu-r/ opb/rep/R-REP-M.2412-2017-PDF-E.pdf.

[47] Z. Zhang, Y. Xiao, Z. Ma et al., "6G wireless networks: vision, requirements, architecture, and key technologies," IEEE Vehicular Technology Magazine, vol. 14, no. 3, pp. 28-41, 2019.

[48] S. Chen, Y. Liang, S. Sun, S. Kang, W. Cheng, and M. Peng, "Vision, requirements, and technology trend of 6G: how to tackle the challenges of system coverage, capacity, user datarate and movement speed," IEEE Wireless Communications, vol. 27, no. 2, pp. 218-228, 2020.

[49] C.-X. Wang, F. Haider, X. Gao et al., "Cellular architecture and key technologies for $5 \mathrm{G}$ wireless communication networks," IEEE Communications Magazine, vol. 52, no. 2, pp. 122-130, 2014.

[50] G. Auer, V. Giannini, C. Desset et al., "How much energy is needed to run a wireless network?," IEEE Wireless Communications, vol. 18, no. 5, pp. 40-49, 2011. 